Publ. RIMS, Kyoto Univ.

23 (1987), 841-879

\title{
Hodge Modules, Equivariant K-Theory and Hecke Algebras
}

By

\author{
Toshiyuki TANISAKI*
}

\section{§ 0. Introduction}

0.1. The Hecke algebra $H(W)$ of a Coxeter system $(W, S)$ is an algebra over the Laurent polynomial ring $\mathbb{Z}\left[q, q^{-1}\right]$ which has a free basis $\left\{T_{w} \mid w \in W\right\}$ and satisfies the following relations :

$$
\begin{array}{ll}
\left(T_{s}+1\right)\left(T_{s}-q\right)=0 & (s \in S) \\
T_{w_{1}} T_{w_{2}}=T_{w_{1}} T_{w_{2}} & \left(l\left(w_{1}\right)+l\left(w_{2}\right)=l\left(w_{1} w_{2}\right)\right),
\end{array}
$$

where $l$ is the length function.

When $W$ is a Weyl group, this algebra appeared in connection with finite Chevalley groups ([I]) as we formulate in the following. Let $G$ be a connected reductive algebraic group with Weyl group $W$ defined and split over a finite field $\mathbb{F}_{q_{0}}$ and $X$ the flag variety of $G$. We denote by $H$ the $\mathbb{C}$ vector space consisting of $\mathbb{C}$-valued functions on $X\left(\mathbb{F}_{q_{0}}\right) \times X\left(\mathbb{F} q_{q_{0}}\right)$ which are invariant under the action of $G\left(\mathbb{F}_{q_{0}}\right) . \quad H$ is endowed with an algebra structure via the convolution product:

$$
\left(h_{1} \cdot h_{2}\right)(x, y)=\sum_{z \in X\left(F q_{0}\right)} h_{1}(x, z) h_{2}(z, y),
$$

and it is isomorphic to the $\mathbb{C}$-algebra obtained by tensoring $\mathbb{C}$ to $H(W)$ over $\mathbb{Z}\left[q, q^{-1}\right]$ via the ring homomorphism $\mathbb{Z}\left[q, q^{-1}\right] \rightarrow \mathbb{C}\left(q \rightarrow q_{0}\right)$.

Replacing functions on $X\left(\mathbb{F}_{q_{0}}\right) \times X\left(\mathbb{F}_{q_{0}}\right)$ by $\mathbb{Q}_{l}$-sheaves on $X \times X$, we have a more sophisticated realization of the Hecke algebra (due to Beilin-

Communicated by M. Kashiwara, March 13, 1987

* Mathematical Institute, Tohoku University, Sendai 980, Japan. 
son, Brylinski and Lusztig-Vogan [LV]). Let $\mathcal{C}$ be the category consisting of constructible $\mathbb{Q}_{l}$-sheaves on $X \times X$, whose restrictions to each $G$-orbit $Y$ have composition factors of the type $\mathbb{Q}_{l, Y}(n)(n \in \mathbb{Z})$, where $(n)$ is the Tate twist. The Grothendieck group $K(C)$ is a $\mathbb{Z}\left[q, q^{-1}\right]$-module via $q^{n}[K]=$ $[K(-n)]$. We define $p_{13}: X \times X \times X \rightarrow X \times X$ and $\mathrm{r}: X \times X \times X \rightarrow X \times X \times$ $X \times X$ by $p_{13}(a, b, c)=(a, c)$ and $r(a, b, c)=(a, b, b, c)$. By the product :

$$
\left[K_{1}\right] \cdot\left[K_{2}\right]=\sum_{j}(-1)^{j}\left[R^{j} p_{13 !}\left(r^{*}\left(K_{1} \rrbracket K_{2}\right)\right)\right],
$$

$K(C)$ is endowed with a $\mathbb{Z}\left[q, q^{-1}\right]$-algebra structure and it is isomorphic to $H(W)$.

0.2. It has been conjectured that there exists a theory in char $=0$, which corresponds to the theory of the weights for $\mathbb{Q}_{l}$-sheaves in char $>0$ (Deligne's philosophy, $[\mathrm{Br}]$ etc.). This was realized by M. Saito as a theory of Hodge modules quite recently $([\mathrm{Sa} 1 \sim 5])$. He has defined, for a non-singular algebraic variety $Y$ over $\mathbb{C}$, a certain abelian category $M H M(Y)$, which is a full subcategory of the category consisting of quartets $(\mathcal{M}, F, K, W)$, where $\mathscr{M}$ is a regular holonomic $D_{Y}$-module, $F$ is a good filtration of $\mathscr{M}, K$ is a perverse sheaf over $\mathbb{Q}$ on $Y$ such that $D R(\mathscr{M})=$ $\mathbb{C} \otimes K$ and $W$ is a filtration of $(\mathcal{M}, F, K)$. This category corresponds to the category of mixed perverse sheaves in char $>0$, philosophically.

Using this theory we can give a realization of $H(W)$ in char $=0$. Let $G$ be a connected reductive algebraic group over $\mathbb{C}$ whose Weyl group is $W$ and let $X$ be the flag variety of $G$. Then there exists a certain abelian category $\mathcal{A}$, which is a subcategory of the category consisting of the objects of $M H M(X \times X)$ with $G$-actions, so that its Grothendieck group $K(\mathcal{l})$ has two free bases $\left\{\left[\mathscr{M}_{w}\right] \mid w \in W\right\}$ and $\left\{\left[\mathcal{L}_{w}\right] \mid w \in W\right\}$ over $\mathbb{Z}\left[q, q^{-1}\right]$ (see Section 3). Here $\mathscr{M}_{w}$ and $\mathcal{L}_{w}$ are certain specified objects of $\mathcal{A}$ and the $\mathbb{Z}\left[q, q^{-1}\right]$-module structure is given by $q^{n}[\mathcal{C V}]=[\mathcal{C V}(-n)]$, where $(n)$ is the counterpart of the Tate twist (see Section 1). Define $p_{13}$ and $r$ similarly to the case of char $>0$. We can show that $\left(\mathcal{H}^{j} p_{13 !}\right)\left(\mathcal{H}^{-\operatorname{dim} x} \boldsymbol{r}^{*}\right)\left(\mathcal{C} \mathcal{V}_{1} \rrbracket \subset V_{2}\right)$ $\in \mathcal{A}$ for $C V_{1}, \mathcal{V}_{2} \in \mathcal{A}$ and a $\mathbb{Z}\left[q, q^{-1}\right]$-algebra structure on $K(\underline{A})$ is defined by :

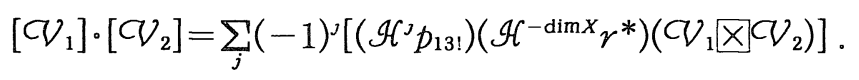

Theorem $\mathbb{A}_{\text {。 }} K(\mathcal{A})$ is isomorphic to $H(W)$ as a $\mathbb{Z}\left[q, q^{-1}\right]$-algebra. 
The isomorphism is given by:

$$
\left[\mathcal{M}_{w}\right] \leftrightarrow(-1)^{\ell(w)} T_{w} \quad \text { and } \quad\left[\mathcal{L}_{w}\right] \leftrightarrow(-1)^{l(w)} \sum_{y \leqq w} F_{y, w}(q) T_{y}
$$

where $P_{y, w}(q)$ are the Kazhdan-Lusztig polynomials (see [KL1]).

D.3. Recently Kazhdan-Lusztig [KL4] and Ginsburg [G2] have given a classification of the irreducible representations of the Hecke algebra of the affine Weyl group $W_{a}$ using equivariant $K$-theory (conjecture of DeligneLanglands-Lusztig). The first step of their work was to define an $\left(H\left(W_{a}\right)\right.$, $H\left(W_{a}\right)$ )-bimodule structure on the equivariant $K$-homology group $K^{G \times C^{*}}(Z)$ of the variety

$$
Z=\left\{(x, y, A) \in X \times X \times \operatorname{Lie}(G) \mid A \text { is nilpotent }, \quad A \in \operatorname{Lie}\left(B_{x}\right) \cap \operatorname{Lie}\left(B_{y}\right)\right\}
$$

and to show that this coincides with the two-sided regular representation of $H\left(W_{a}\right)$. Here $B_{x}$ is the Borel subgroup of $G$ corresponding to $x \in X$. We review this briefly following the formulation of Ginsburg. Let $p: T^{*} X \rightarrow$ $X$ be the cotangent bundle. Regarding $Z$ as a subvariety of $T^{*}(X \times X)=$ $T^{*} X \times T^{*} X$, we can view $K^{G \times C *}(Z)$ as the Grothendieck group of the abelian category consisting of coherent $O_{T: X \times T^{*} X}$-modules with $G \times \mathbb{C}^{*}$. actions supported in $Z$. Note that $K^{G \times C^{*}}(Z)$ is a $\mathbb{Z}\left[q, q^{-1}\right]$-module since the representation ring of $\mathbb{C}^{*}$ is identified with $\mathbb{Z}\left[q, q^{-1}\right]$. Lat $p_{\imath}: T^{*} X \times$ $T^{*} X \times T^{*} X \rightarrow T^{*} X \times T^{*} X$ and $p_{2}: T^{*} X \times T^{*} X \times T^{*} X \rightarrow T^{*} X$ be the obvious projections. It is easily seen that a $\mathbb{Z}\left[q, q^{-1}\right]$-algebra structure on $K^{G \times C^{*}}(Z)$ is defined by :

$$
\left[M_{1}\right] \cdot\left[M_{2}\right]=\sum_{j}(-1)^{\jmath}\left[\mathcal{H}^{\jmath}\left(\mathbb{R} p_{13 *}\left(p_{12} * M_{1} \stackrel{L}{\otimes} p_{23} * M_{2} \stackrel{L}{\otimes} p_{2}^{*} p^{*} \Omega_{X}\right)\right)\right],
$$

where $\Omega_{X}$ is the sheaf of the differential forms of the highest degree on $X$. The result is that this algebra is isomorphic to $H\left(W_{a}\right)$, especially isomorphic to the two-sided regular representation as an $\left(H\left(W_{a}\right), H\left(W_{a}\right)\right)$ bimodule (see Section 4.2 for the explicit description of the isomorphism).

0.4. Let $C V=(\mathcal{M}, F, K, W)$ be an object of $\mathcal{A}$. Then $\operatorname{Gr}^{F} \mathcal{M}$ is a coherent module over the $O_{X \times X}$-algebra $\operatorname{Gr}^{F} D_{X \times X}=(p \times p)_{*}\left(O_{T^{*} X \times T^{*} X}\right)$. Hence

$$
\operatorname{gr} C V=O_{T^{*} \times \times T^{\star} X} \bigotimes_{(p \times p)^{-1}\left(\mathrm{Gr}^{F} D_{X \times X}\right)}(p \times p)^{-1}\left(\mathrm{Gr}^{F} \mathcal{M}\right)
$$

is a coherent $O_{T^{*} X \times T^{*} X}$-module with $G \times \mathbb{C}^{*}$-action. It is easily seen that the support of gr $C V$ is contained in the union $\Lambda$ of the conormal bundles of 
the $G$-orbits on $X \times X$. We define an involution $a$ on $T^{*} X \times T^{*} X$ by $a(x$, $y, \xi, \eta)=(x, y, \xi,-\eta)$, where $(x, y)$ is a coordinate of $X \times X$ and $(\xi, \eta)$ is a coordinate of fibers. Since $a(\Lambda)=Z$, we have a $\mathbb{Z}\left[q, q^{-1}\right]$-module homomorphism :

$$
\gamma=q^{\operatorname{dim} X}\left(a^{*} \circ \mathrm{gr}\right): K(\mathfrak{A}) \rightarrow K^{G \times C^{*}}(Z) .
$$

Theorem B. $\gamma$ is a homomorphism of $\mathbb{Z}\left[q, q^{-1}\right]$-algebra, and when we identify $K(\mathcal{A})$ and $K^{G \times c^{*}}(Z)$ with $H(W)$ and $H\left(W_{a}\right)$ respectively, $\gamma$ coincides with the natural inclusion.

The main difficulty in proving $K^{G \times C^{*}}(Z) \simeq H\left(W_{a}\right)$ is to show that the action of $H(W)$ is well-defined. Ginsburg and Kazhdan-Lusztig used the localization theorem in equivariant $K$-theory and reduced the problem to the case of $K^{G \times C^{*}}(X \times X)$. Then the problem turned out to be a combinatorial one, which had been already solved in [Lu] (see also Kato's simpler solution given in [KL4]). In a sence Theorem $B$ gives a different proof of this fact. Although our proof relies on the deep theory of Hodge modules, it seems that it gives a more natural explanation of the fact that the Hecke algebra appears in the context of equivariant $K$-theory.

0.5. The contents of this paper are as follows. In Section 1 we give a brief summary of the theory of Hodge modules and state some facts concerning the Hodge modules with group actions. In Section 2 we review the definition of the equivariant $K$-homology groups and give some relation between Hodge modules with group actions and equivariant $K$-theory. In Sections 3 and 4 Theorem $A$ and Theorem $B$ are proved, respectively. In Section 5 we treat some problems concerning good filtrations of the modules over the enveloping algebra of the Lie algebra of $G$ associated to Hodge modules.

In Sections 1 and 2 the letters $G$ and $X$ will be used for a general algebraic group and a general algebraic variety, respectively, while in Sections 3 to 5 they will be used for a connected reductive algebraic group and its flag variety, respectively. The letter $W$ is used for both of the Weyl group and the weight filtration. We hope that readers will distinguish them from the context.

0.6. The author would like to express his deep gratitude to M. Saito for explaining to him the theory of Hodge modules including results which are not yet written down and answering (sometimes trivial) questions 
concerning them. He would also like to thank R. Hotta and M. Kashiwara for useful conversation.

Although the author had to wait for the theory of Hodge modules, he got the main idea of this paper while he was staying at Harvard University in 1984. He would like to thank for its hospitality. He also thanks the comitee on Educational Project for Japanese Mathematical Scientists for supporting the stay.

\section{$\S \mathbb{1}$. Hodge Modules}

\subsection{Hodge structures (see [D2])}

We recall basic notions concerning Hodge structures.

Let $H$ be a finite dimensional vector space over $\mathbb{Q}$ and $F$ a decreasing filtration of $H_{C}=\mathbb{C} \otimes_{Q} H$. Hence $F^{p}\left(H_{C}\right)$ is a $\mathbb{C}$-subspace of $H_{C}$ for each $p \in \mathbb{Z}, F^{p}\left(H_{c}\right) \cap F^{p+1}\left(H_{c}\right), F^{p}\left(H_{c}\right)=0$ for a sufficiently large $p$ and $F^{p}\left(H_{C}\right)=H_{C}$ for a sufficiently small $p . \quad(H, F)$ is called a Hodge structure of weight $n$ if $H_{C}=F^{p} \oplus \bar{F}^{n-p+1}$ for any $p$. Here barring denotes the complex conjugate. Setting $H^{p, q}=F^{p} \cap \bar{F}^{q}$ we have the Hodge decomposition $H_{C}=\oplus_{p} H^{p, n-p}$. When $(H, F)$ and $\left(H^{\prime}, F^{\prime}\right)$ are Hodge structures of weight $n$, a linear map $f: H \rightarrow H^{\prime}$ is called a morphism (of Hodge structures of weight $n)$ if $f\left(F^{p}\right) \subset F^{\prime p}$ for any $p$. We denote the category of Hodge structures of weight $n$ by $S H(n)$.

A polarization of $(H, F) \in S H(n)$ is a bilinear form $S$ on $H$, which is symmetric (resp. skew symmetric) if $n$ is even (resp. odd) and satisfies the following condition:

$$
\begin{aligned}
& S\left(H^{p, n-p}, H^{p^{\prime}, n-p^{\prime}}\right)=0 \quad \text { unless } \quad p+p^{\prime}=n, \\
& (\sqrt{-1})^{n-2 p} S(v, \bar{v})>0 \quad \text { for } \quad v \in H^{p, n-p}, v \neq 0 .
\end{aligned}
$$

$(H, F) \in S H(n)$ is said to be polarizable if there exists a polarization of ( $H$, $F)$. We denote the full subcategory of $S H(n)$ consisting of polarizable Hodge structures by $S H(n)^{p}$. It is a semisimple abelian category.

Let $H$ be a finite dimensional Q -vector space, $F$ a decreasing filtration of $\mathbb{C} \underset{Q}{\otimes} H$ and $W=\left\{W_{n}\right\}$ an increasing filtration of $(H, F)$. Then $(H, F, W)$ is called a mixed Hodge structure if $\mathrm{Gr}_{n}{ }^{W}(H, F)\left(=W_{n}(H, F) / W_{n-1}(H, F)\right)$ $\in S H(n)$ for any $n$. The category SHM of mixed Hodge structures is 
defined similarly. We denote by $S H M^{p}$ the full subcategory of $S H M$ consisting of $(H, F, W)$ with $\operatorname{Gr}_{n}{ }^{W}(H, F) \in S H(n)^{p}$ for any $n$.

Let $X$ be a non-singular algebraic variety over $\mathbb{C}$. We denote the sheaf of algebraic differential operators on $X$ by $D_{X}$. Let $H$ be a $\mathbb{Q}$-local system on $X$. Hence $H$ is a sheaf of $\boldsymbol{Q}$-vector spaces on the associated complex manifold $X_{a n}$ (in the classical topology) which is locally constant and has finite dimensional stalks. By the Riemann-Hilbert correspondence for local systems due to Deligne [D1] there exists a unique regular holonomic $D_{X}$-module $\mathscr{M}(H)$ which is locally free as an $O_{X}$-module and satisfies $\mathcal{M}(H)_{a n} \simeq O_{X_{a n}} \otimes Q_{Q} H$. Here $O_{X}$ is the structure sheaf of $X, O_{X_{a n}}$ is the sheaf of holomorphic functions on $X_{a n}, D_{X_{a n}}=O_{X_{a n}} \otimes_{O_{X}} D_{X}$ and $\mathcal{M}(H)_{a n}=$ $O_{X_{a n}} \otimes_{O_{x}} \mathcal{M}(H)=D_{X_{a n}} \otimes_{D_{x}} \mathscr{M}(H)$. Let $F$ be a decreasing filtration of $\mathcal{M}(H)$ by $O_{X}$-submodules such that $F^{p}(\mathscr{M}(H)) / F^{p+1}(\mathscr{M}(H))$ is locally free for any $p$. Then $(H, F)$ is called a variation of Hodge structures of weight $n$ if $\left(H_{x}\right.$, $F(x)) \in S H(n)$ for any $x \in X$ and $\partial \cdot F^{p}(\mathscr{M}(H)) \subset F^{p-1}(\mathscr{M}(H))$ for any vector field $\partial$ and any $p$. Note that the fiber of $\mathcal{M}(H)$ at $x \in X$ is $\underset{Q}{\mathbb{C}} \otimes_{Q} H_{x}$ and $F$ induces a filtration $F(x)$ of $\underset{Q}{\mathbb{Q}} H_{x}$. The category of variations of Hodge structures of weight $n$ is denoted by $\operatorname{VSH}(X, n)$. A polarization of $(H, F) \in V S H(X, n)$ is a $\mathbb{Q}_{X}$-linear map $H \underset{\mathbb{Q}_{x}}{\bigotimes_{X} H \rightarrow \mathbb{Q}_{X}}$ which gives a polarization of $\left(H_{x}, F(x)\right)$ for any $x \in X$. The full subcategory of $\operatorname{VSH}(X, n)$ consisting $(H, F) \in V S H(X, n)$ which are polarizable is denoted by $\operatorname{VSH}(X, n)^{p}$. Categories $\operatorname{VSHM}(X)$ and $\operatorname{VSHM}(X)^{p}$ are defined similarly to $S H M$ and $S H M^{p}$, respectively.

If $f: X \rightarrow Y$ is a morphism of non-singular varieties, we have natural functors $\operatorname{VSH}(Y, n) \rightarrow \operatorname{VSH}(X, n), \operatorname{VSH}(Y, n)^{p} \rightarrow \operatorname{VSH}(X, n)^{p}, \operatorname{VSHM}(Y)$ $\rightarrow \operatorname{VSHM}(X)$ and $\operatorname{VSHM}(Y)^{p} \rightarrow \operatorname{VSHM}(X)^{p}$. All of them are denoted by $f^{*}$.

1.2. Filtered $\mathbb{D}$-modules and functors (see [Be], [Sa2; Section 2])

For a non-singular algebraic variety $X$ over $\mathbb{C}$ let $M_{r h}\left(D_{X}\right)$ be the category of regular holonomic $D_{X}$-modules. Since we are working in the algebraic category, the regularity here includes the regularity at infinity (see $[\mathrm{Be}]$ ).

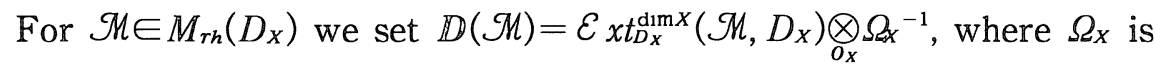


the sheaf of differential forms of degree $\operatorname{dim} X$. It is known that $\mathcal{E}_{x} t_{D_{X}}^{i}(\mathcal{M}$, $\left.D_{X}\right)=0$ for $i \neq \operatorname{dim} X$ and $\mathbb{D}(\mathscr{M})$ is a regular holonomic $D_{X}$-module. More generally, for a bounded complex $\mathscr{M}$ of $D_{X}$-modules such that $\mathscr{H}^{i}(\mathscr{M}) \in$ $M_{T h}\left(D_{X}\right)$ for each $i$, we set:

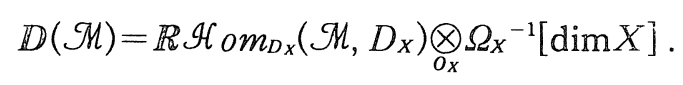

Let $f: X \rightarrow Y$ be a morphism of non-singular varieties. An $\left(f^{-1} D_{Y}\right.$, $D_{X}$ )-bimodule $D_{Y \leftarrow X}$ and a $\left(D_{X}, f^{-1} D_{Y}\right)$-bimodule $D_{X \rightarrow Y}$ are defined by:

$$
D_{Y \leftarrow X}=f^{-1}\left(D_{Y} \underset{O_{Y}}{\otimes} \Omega_{Y}{ }^{-1}\right) \underset{f^{-1} O_{Y}}{\otimes} \Omega_{X}, \quad D_{X \rightarrow Y}=O_{X} \underset{f^{-1} O_{Y}}{\otimes} f^{-1} D_{Y} .
$$

Then for each $j \in \mathbb{Z}$ additive functors :

$$
\begin{aligned}
& \mathscr{H}^{j} f_{*} \text { and } \mathscr{M}^{j} f_{1}: M_{r h}\left(D_{X}\right) \rightarrow M_{r h}\left(D_{Y}\right) \text {, } \\
& \mathscr{H}^{j} f^{\prime} \text { and } \mathscr{H}^{j} f^{*}: M_{r h}\left(D_{Y}\right) \rightarrow M_{r h}\left(D_{X}\right)
\end{aligned}
$$

are defined as the $j$-th cohomologies of the functors $f_{*}, f_{1}, f^{\prime}, f^{*}$ between derived categories given by:

$$
\begin{aligned}
& f_{*}(\mathcal{M})=\mathbb{R} f_{*}\left(D_{Y \leftarrow X} \underset{D_{X}}{\otimes} \mathcal{M}\right), \quad f_{1}(\mathscr{M})=\mathbb{D}\left(f_{*}(\mathbb{D}(\mathcal{M}))\right), \\
& f^{\prime}(\mathscr{M})=\left(D_{X \rightarrow Y} \underset{f^{-1} D_{Y}}{\stackrel{L}{\otimes}} f^{-1} \mathscr{M}\right)[\operatorname{dim} X-\operatorname{dim} Y], \quad f^{*}(\mathscr{M})=\mathbb{D}\left(f^{\prime}(\mathbb{D}(\mathscr{M}))\right) .
\end{aligned}
$$

We have a natural increasing filtration $F$ of $D_{X}$ given by the orders of differential operators. If an increasing filtration $F$ of a $D_{X}$-module $\mathscr{M}$ by $O_{X}$-submodules satisfies the conditions:

$$
\begin{aligned}
& F_{p}\left(D_{X}\right) F_{q}(\mathscr{M}) \subset F_{p+q}(\mathscr{M}) \text { for any } p, q \in \mathbb{Z}, \\
& \mathscr{M}=\bigcup_{p} F_{p}(\mathscr{M}), \\
& F_{p}(\mathscr{M})=0 \text { for a sufficiently small } p,
\end{aligned}
$$

then $(\mathscr{M}, F)$ is called a filtered $D_{X}$-module. When $\mathrm{Gr}^{F} \mathscr{M}$ is a coherent $\mathrm{Gr}^{F} D_{X}$-module, $F$ is called a good filtration. Let $M F_{r h}\left(D_{X}\right)$ be the category consisting of filtered $D_{X}$-module $(\mathcal{M}, F)$ such that $\mathcal{M}$ is regular holonomic and $F$ is a good filtration. This is not an abelian category but an exact category.

For a projective morphism $f: X \rightarrow Y$ and $(\mathcal{M}, F) \in M F_{r h}\left(D_{X}\right)$, an object $f_{*}(\mathcal{M}, F)$ of the derived category consisting of complexes of filtered $D_{Y}$. modules is defined (see [Sa2: Section 2]). Forgetting the filtration this 


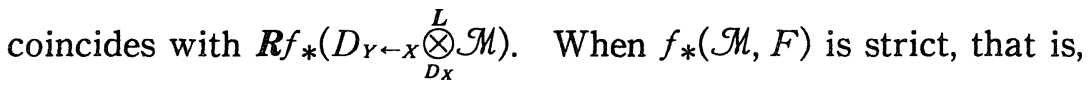

$$
\mathscr{H}^{j}\left(F_{p}\left(f_{*}(\mathscr{M}, F)\right)\right) \rightarrow \mathscr{Y}^{j}\left(\boldsymbol{R} f_{*}\left(D_{Y \leftarrow X} \stackrel{L}{\otimes} \mathscr{M}\right)\right)\left(=\mathscr{H}^{j} f_{*}(\mathscr{M})\right)
$$

is injective for any $j$ and $p$, a good filtration of $\mathscr{H}^{j} f_{*}(\mathscr{M})$ is given by $F_{p}\left(\mathscr{H}^{j} f_{*}(\mathscr{M})\right)=\mathscr{H}^{j}\left(F_{p}\left(f_{*}(\mathscr{M}, F)\right)\right)$. This object of $M F_{r h}\left(D_{Y}\right)$ is denoted by $\mathcal{H}^{j} f_{*}(M, F)$.

When $f$ is a closed immersion, $\mathscr{H}^{j} f_{*}(\mathscr{M})=0$ for $j \neq 0$ and $f_{*}(\mathscr{M}, F)$ is always strict. $f_{*}(\mathscr{M}, F)\left(=\mathscr{H}^{o} f_{*}(\mathscr{M}, F)=\left(f_{*}\left(D_{Y \leftarrow x} \otimes \mathscr{M}\right), F\right)\right)$ is given by :

$$
F_{p}\left(f_{*}\left(D_{Y \leftarrow X} \otimes \mathscr{M}\right)\right)=f_{*}\left(\sum_{q} F_{q}\left(D_{Y \leftarrow X}\right) \otimes F_{p-q+\operatorname{dim} X-\operatorname{dim} Y}(\mathcal{M})\right),
$$

where the filtration of $D_{Y \leftarrow X}$ is induced from that of $D_{Y}$.

When $X=Y \times Z$ and $f: X \rightarrow Y$ is the projection ( $Z$ is a projective non-singular variety with dimension $m), D_{Y \leftarrow X} \bigotimes_{D_{X}}^{L} \mathcal{M}$ is quasi-isomorphic to the relative de Rham complex :

$$
D R_{X / Y}(\mathcal{M})=\left[\Omega_{X / Y}^{0} \bigotimes_{O_{X}} \mathcal{M} \rightarrow \Omega_{X / Y}^{1} \bigotimes_{O_{X}} \mathcal{M} \rightarrow \cdots \rightarrow \Omega_{X / Y}^{m} \bigotimes_{O_{X}} \mathcal{M}\right],
$$

where $\Omega_{X / Y}^{i}$ is the sheaf of relative differential forms of degree $i$ and the last term $\Omega_{X / Y}^{m} \otimes \mathcal{M}$ has the complex degree 0 . With the filtration:

$$
F_{p}\left(D R_{X / Y}(\mathscr{M})\right)=\left[\Omega_{X / Y}^{0} \underset{O_{X}}{\otimes} F_{p}(\mathcal{M}) \rightarrow \cdots \rightarrow \Omega_{X / Y}^{m} \underset{O_{X}}{\otimes} F_{p+m}(\mathcal{M})\right],
$$

$D R_{X / Y}(\mathcal{M}, F)=\left(D R_{X / Y}(\mathcal{M}), F\right)$ is a complex of filtered $f^{-1} D_{Y}$-modules. Then $f_{*}(\mathcal{M}, F)$ is strict if and only if the homomorphism $\mathscr{H}^{j}\left(\mathbb{R} f_{*}\left(F_{p}\left(D R_{X / Y}(\mathscr{M})\right)\right)\right) \rightarrow \mathscr{H}^{j}\left(\boldsymbol{R} f_{*}\left(D R_{X / Y}(\mathscr{M})\right)\right)\left(=\mathscr{H}^{j} f_{*}(\mathscr{M})\right)$ is injective for any $j$ and $p$, and in this case $\mathscr{H}^{j} f_{*}(\mathscr{M}, F)$ is given by $F_{p}\left(\mathscr{H}^{j} f_{*}(\mathscr{M})\right)=$ $\mathscr{H}^{j}\left(\mathbb{R} f_{*}\left(F_{p}\left(D R_{X / Y}(\mathscr{M})\right)\right)\right)$.

Example. Let $f: X \rightarrow Y$ be a $\boldsymbol{P}^{1}$-bundle. We define a good filtration of $O_{X}$ and $O_{Y}$ by $\mathrm{Gr}_{j}{ }^{F} O_{X}=0$ and $\mathrm{Gr}_{j}{ }^{F} O_{Y}=0$ for $j \neq 0$. Then it is easily seen that $f_{*}\left(O_{X}, F\right)$ is strict and $\mathcal{H}^{j} f_{*}\left(O_{X}, F\right)=0$ for $j \neq \pm 1, \mathscr{H}^{-1} f_{*}\left(O_{X}, F\right)$ $=\left(O_{Y}, F\right)$ and $\mathscr{H}^{1} f_{*}\left(O_{X}, F\right)=\left(O_{Y}, F[-1]\right)$. For an increasing filtration $F$ and $n \in \mathbb{Z}, F[n]$ is a new filtration given by $F[n]_{p}=F_{p-n}$.

\section{3̊. Pure Hodge modules}

Let $X$ be a non-singular algebraic variety over $\mathbb{C}$. We denote by $\operatorname{Perv}\left(\mathbb{C}_{X}\right)$ (resp. Perv $\left(\mathbb{Q}_{X}\right)$ ) the abelian category of perverse sheaves over $\mathbb{C}$ (resp. $\mathbb{Q})$ on $X([\mathrm{BBD}])$. For a regular holonomic $D_{X}$-module $\mathscr{M}$ 


$$
D R_{X}(\mathcal{M})=\mathbb{R} \mathcal{H} \operatorname{Som}_{D_{x_{a n}}}\left(O_{X_{a n}}, D_{X_{a n}} \bigotimes_{D_{X}} \mathcal{M}\right)[\operatorname{dim} X]
$$

belongs to $\operatorname{Perv}\left(\mathbb{C}_{X}\right)$ and the functor:

$$
D R_{X}: M_{r h}\left(D_{X}\right) \rightarrow \operatorname{Perv}\left(\mathbb{C}_{X}\right)
$$

gives an equivalence of abelian categories (the Riemann-Hilbert correspondence, $[\mathrm{K}],[\mathrm{Me} 1,2]$, see also $[\mathrm{Be}]$ for the algebraic version stated above). It is known that the functor $D R_{X}$ is compatible with direct images and inverse images, that is, we have:

$$
\begin{aligned}
& D R_{Y} \circ\left(\mathcal{H}^{j} f_{*}\right)=\left({ }^{p} \mathcal{H}^{j} f_{*}\right) \circ D R_{X}, \quad D R_{Y} \circ\left(\mathcal{H}^{j} f_{!}\right)=\left({ }^{p} \mathcal{H}^{\jmath} f_{!}\right) \circ D R_{X}, \\
& D R_{X} \circ\left(\mathcal{H}^{j} f^{\prime}\right)=\left({ }^{p} \mathcal{H}^{j} f^{!}\right) \circ D R_{Y}, \quad D R_{X} \circ\left(\mathcal{H}^{j} f^{*}\right)=\left({ }^{p} \mathcal{H}^{j} f^{*}\right) \circ D R_{Y},
\end{aligned}
$$

where ${ }^{p} \mathcal{H}^{j}$ is the perverse cohomology.

Let $M F_{r h}\left(D_{X}, \mathbb{Q}\right)$ be the fiber product of the categories $M F_{r h}\left(D_{X}\right)$ and $\operatorname{Perv}\left(\mathbb{Q}_{X}\right)$ over $\operatorname{Perv}\left(\mathbb{C}_{X}\right)$. An object of $M F_{r h}\left(D_{X}, \mathbb{Q}\right)$ is a triple $(\mathcal{M}, F, K)$, where $\mathcal{M}$ is a regular holonomic $D_{X}$-module, $F$ is a good filtration of $\mathcal{M}$ and $K$ is a perverse sheaf over with a given isomorphism $D R(\mathcal{M}) \simeq \mathbb{C}$ $\bigotimes_{Q} K . \quad$ A fully faithful functor :

$$
\phi_{X}{ }^{n}: \operatorname{VSH}(X, n) \rightarrow M F_{r h}\left(D_{X}, \mathbb{Q}\right)
$$

is defined by :

$$
\phi_{X}{ }^{n}(H, F)=(\mathcal{M}(H), F, H[\operatorname{dim} X]) \text { with } \quad F_{p}=F^{-p}
$$

(see Section 1.1).

In [Sa1, 2] certain full subcategories $M H(X, k)^{p}$ and $M H_{Z}(X, k)^{p}$ of $M F_{r h}\left(D_{X}, Q\right)$ are defined. Here $k$ is an integer and $Z$ is an irreducible closed subvariety of $X$. We do not reproduce their definitions but list some properties which will be used later.

(p1) $M H(X, k)^{p}$ and $M H_{Z}(X, k)^{p}$ are abelian categories whose morphisms are always strict with respect to $F$.

(p2) $M H(X, k)^{p}=\bigoplus_{Z} M H_{Z}(X, k)^{p}$. That is, any object of $M H(X, k)^{p}$ is decomposed uniquely into the direct sum of the objects of $M H_{Z}(X, k)^{p}$, and if $C V_{2} \in M H_{Z_{z}}(X, k)^{p}(i=1,2)$ with $Z_{1} \neq Z_{2}$, then $\operatorname{Hom}\left(C V_{1}, C V_{2}\right)=0$.

(p3) Let $X$ be the union of open subsets $U_{\lambda}$ and let $C V \in M F_{r h}\left(D_{X}, \mathbb{Q}\right)$. Then $C \in M H(X, k)^{p}$ if and only if $C V \mid U_{\lambda} \in M H\left(U_{\lambda}, k\right)^{p}$ for any $\lambda$.

(p4) If $C V=(\mathscr{M}, F, K) \in M H(X, k)^{p}$, then 


$$
\mathcal{V}(n)=(\underset{\mathbb{Q}}{\mathbb{Q}}(n), F[n], K \underset{\mathbb{Q}}{\otimes} \mathbb{Q}(n)) \in M H(X, k-2 n)^{p},
$$

where $Q(n)=(2 \pi \sqrt{-1})^{n} \mathbb{Q} \subset \boldsymbol{C}$ and $F[n]_{p}=F_{p-n}$.

(p5) $\phi_{X}{ }^{n}(H, F)$ belongs to $M H_{X}(X, n+\operatorname{dim} X)^{p}$ for $(H, F) \in V S H(X$, $n)^{p}$. Especially

$$
\mathcal{L}_{X}=\left(O_{X}, F, \mathbb{Q}_{X}[\operatorname{dim} X]\right) \text { with } \operatorname{Gr}_{i}{ }^{F} O_{X}=0 \text { for } i \neq 0
$$

is an object of $M H(X, \operatorname{dim} X)^{p}$.

(p6) For an object $(\mathcal{M}, F, K)$ of $M H_{Z}(X, k)^{p}$, there exist a nonsingular open subset $U$ of $Z\left(Y=Z-U, i: Z \hookrightarrow X, i_{0}: U \hookrightarrow X-Y\right)$ and $(H$, $F) \in V S H(U, k-\operatorname{dim} Z)^{p}$ such that $K=i_{*} \mathcal{I C}(H)$ and $(\mathcal{M}, F, K) \mid X-Y=$ $\left(i_{0 *}(\mathscr{M}(H), F), i_{0 *} H[\operatorname{dim} Z]\right)$. Here $\mathcal{I C}(H)$ is the $D G M$-extension of $H$ (see [GM], [BBD]).

(p7) Let $(\mathscr{M}, F, K)$ and $\left(\mathscr{M}^{\prime}, F^{\prime}, K^{\prime}\right)$ be objects of $M H_{Z}(X, k)^{p}$. Choose a non-singular open subset $U$ of $Z$ and $(H, F),\left(H^{\prime}, F^{\prime}\right) \in V S H(U$, $k-\operatorname{dim} Z)^{p}$ so that $U$ and $(H, F)\left(\right.$ resp. $\left.\left(H^{\prime}, F^{\prime}\right)\right)$ satisfy the conclusion of (p6) for $(\mathcal{M}, F, K)$ (resp. $\left.\left(\mathscr{M}^{\prime}, F^{\prime}, K^{\prime}\right)\right)$. Then any morphism from $(H, F)$ to $\left(H^{\prime}, F^{\prime}\right)$ in $V S H(U, k-\operatorname{dim} Z)^{p}$ extends uniquely to a morphism from $(\mathscr{M}, F, K)$ to $\left(\mathscr{M}^{\prime}, F^{\prime}, K^{\prime}\right)$ in $M H_{Z}(X, k)^{p}$. Especially $(\mathscr{M}, F, K)$ in $(\mathrm{p} 6)$ is uniquely determined by $U$ and $(H, F)$.

(p8) For a projective morphism $f: X \rightarrow Y$ of non-singular varieties $f_{*}(\mathcal{M}, F)$ is strict for $\mathcal{C}=(\mathcal{M}, F, K) \in M H(X, k)^{p}$ and

$$
\mathscr{H}^{j} f_{*}(\mathcal{C V}):=\left(\mathcal{H}^{j} f_{*}(\mathcal{M}, F),{ }^{p} \mathcal{H}^{j} f_{*}(K)\right) \in M H(Y, j+k)^{p} .
$$

(p9) Let $f: X \rightarrow Y$ and $g: Y \rightarrow Z$ be projective morphisms of nonsingular varieties. Then $\mathscr{H}^{j}(g \circ f)_{*}(\mathcal{Z})=\underset{k}{\oplus}\left(\mathcal{H}^{k} g_{*}\right)\left(\mathscr{H}^{j-k} f_{*}\right)(\mathcal{C})$ for $\mathcal{V} \in$ $M H(X, n)^{p}$.

Definition. Let $Z$ be an irreducible closed subvariety of a nonsingular variety $X$ with singular locus $Z_{\text {sing }}$ and natural inclusion $i$ : $Z-Z_{\text {sing }} \rightarrow X-Z_{\text {sing. }}$ It follows from (p2), (p6), (p7) and the desingularization theorem of Hironaka that there exists a unique object $C$ of $M H_{Z}(X$, $\operatorname{dim} Z)^{p}$ such that $\mathcal{V} \mid X-Z_{\text {sing }}=i_{*} \mathcal{L}_{Z-z_{\text {s.ng }}}$. We denote this $\mathcal{Q V}$ by $\mathcal{L}(Z, X)$.

Example. If $f: X \rightarrow Y$ is a $\boldsymbol{P}^{1}$-bundle of non-singular varieties, we have $\mathscr{H}^{j} f_{*}\left(\mathcal{L}_{X}\right)=0$ for $j \neq \pm 1, \mathscr{H}^{-1} f_{*}\left(\mathcal{L}_{X}\right)=\mathcal{L}_{Y}$ and $\mathscr{H}^{1} f_{*}\left(\mathcal{L}_{X}\right)=\mathcal{L}_{Y}(-1)$.

\subsection{Mixed Hodge modules}

For a non-singular variety $X$ let $M H W(X)^{p}$ be the category consisting 
of quartets $(\mathcal{M}, F, K, W)$ where $(\mathscr{M}, F, K)$ is an object of $M F_{r h}\left(D_{X}, \mathbb{Q}\right)$ and $W$ is a finite increasing filtration of $(\mathscr{M}, F, K)$ in $M F_{r h}\left(D_{X}, \mathbb{Q}\right)$ such that $\operatorname{Gr}_{k}{ }^{W}(\mathcal{M}, F, K)$ is an object of $M H(X, k)^{p}$ for any $k$. In view of (p5) we have a natural functor:

$$
\phi_{X}: \operatorname{VSHM}(X)^{p} \rightarrow M H W(X)^{p} .
$$

Saito has defined a certain full subcategory $\operatorname{MHM}(X)$ of $M H W(X)^{p}$ and additive functors :

$$
\mathcal{H}^{j} f_{1}: \operatorname{MHM}(X) \rightarrow \operatorname{MHM}(Y), \quad \mathscr{H}^{j} f^{*}: \operatorname{MHM}(Y) \rightarrow \operatorname{MHM}(X)
$$

for a morphism $f: X \rightarrow Y$ of non-singular varieties $\left(\mathscr{H}^{j} f_{*}\right.$ and $\mathscr{H}^{j} f^{\prime}$ are also defined. But we do not use them.). We list some of their properties in the following ([Sa3 5]).

(m1) $\operatorname{MHM}(X)$ is an abelian category whose morphisms are always strict for both $F$ and $W$.

(m2) $M H M(X)$ is closed under subquotients in $M H W(X)^{p}$.

(m3) If $\mathscr{H}^{j} f_{1}(\mathscr{M}, F, K, W)=\left(\mathscr{M}^{\prime}, F^{\prime}, K^{\prime}, W^{\prime}\right)$, then $\mathscr{M}^{\prime}=\mathscr{H}^{j} f_{1}(\mathscr{M})$ and $K^{\prime}={ }^{p} \mathscr{H}^{\jmath} f_{1}(K)$.

(m4) If $\mathscr{H}^{\jmath} f^{*}(\mathscr{M}, F, K, W)=\left(\mathscr{M}^{\prime}, F^{\prime}, K^{\prime}, W^{\prime}\right)$, then $\mathscr{M}^{\prime}=\mathscr{H}^{\jmath} f^{*}(\mathscr{M})$ and $K^{\prime}={ }^{p} \mathscr{K}^{j} f^{*}(K)$.

(m5) If $\mathcal{C V}=(\mathcal{M}, F, K, W) \in M H M(X)$, then we have :

$$
C \mathcal{V}(n):=(\mathcal{M} \otimes \mathbb{Q}(n), F[n], K \underset{Q}{Q} \mathbb{Q}(n), W[-2 n]) \in M H M(X) .
$$

(m6) For a short exact sequence $0 \rightarrow C V_{1} \rightarrow C V_{2} \rightarrow C V_{3} \rightarrow 0$ in $M H M(X)$ we have a long exact sequence :

$$
\ldots \rightarrow \mathscr{H}^{j} f_{1}\left(C V_{1}\right) \rightarrow \mathscr{H}^{j} f_{1}\left(C V_{2}\right) \rightarrow \mathcal{H}^{j} f_{1}\left(C V_{3}\right) \rightarrow \mathscr{H}^{\jmath+1} f_{1}\left(\mathcal{V}_{1}\right) \rightarrow \ldots
$$

in $M H M(Y)$ which coincides with the usual long exact sequence caused by $\mathscr{H}^{j} f_{1}\left(\right.$ resp. $\left.{ }^{p} \mathcal{H}^{j} f_{1}\right)$ on the level of $M_{r_{h}}\left(D_{Y}\right)$ (resp. $\left.\operatorname{Perv}\left(\mathbb{Q}_{Y}\right)\right)$.

(m7) For a short exact sequence $0 \rightarrow C V_{1} \rightarrow \mathcal{C V}_{2} \rightarrow C V_{3} \rightarrow 0$ in $M H M(Y)$ we have a long exact sequence :

$$
\ldots \rightarrow \mathscr{H}^{j} f^{*}\left(C V_{1}\right) \rightarrow \mathscr{H}^{j} f^{*}\left(C V_{2}\right) \rightarrow \mathscr{H}^{j} f^{*}\left(C V_{3}\right) \rightarrow \mathscr{M}^{\jmath+1} f^{*}\left(C V_{1}\right) \rightarrow \ldots
$$

in $M H M(X)$ which coincides with the usual long exact sequence caused by $\mathscr{H}^{\jmath} f^{*}\left(\right.$ resp. $\left.{ }^{p} \mathscr{H}^{\jmath} f^{*}\right)$ on the level of $M_{r h}\left(D_{X}\right)$ (resp. $\operatorname{Perv}\left(\mathbb{Q}_{X}\right)$ ).

(m8) Let $f: X \rightarrow Y$ be a smooth morphism with relative dimension $m$. (Hence $\mathscr{H}^{\jmath} f^{*}=0$ for $j \neq m$.) Set $\left(\mathscr{H}^{m} f^{*}\right)(\mathscr{M}, F, K, W)=\left(\mathscr{M}^{\prime}, F^{\prime}, K^{\prime}, W^{\prime}\right)$ 
for $(\mathcal{M}, F, K, W) \in M H M(Y)$. Then we have :

$$
\begin{gathered}
\mathscr{M}^{\prime}=O_{X} \underset{f^{-1} O_{Y}}{ } f^{-1} \mathscr{M}, \quad K^{\prime}={ }^{p} \mathscr{K}^{m} f^{*}(K), \quad F_{p}\left(\mathcal{M}^{\prime}\right)=O_{X} \otimes f^{-1}\left(F_{p}(\mathscr{M})\right), \\
W_{q}\left(\mathscr{M}^{\prime}, F^{\prime}, K^{\prime}\right)=\left(\mathscr{M}^{\prime \prime}, F^{\prime \prime}, K^{\prime \prime}\right) \quad \text { with } \\
\mathscr{M}^{\prime \prime}=O_{X} \otimes f^{-1}\left(W_{q-m}(\mathscr{M})\right), \quad K^{\prime \prime}={ }^{p} \mathscr{H}^{m} f^{*}\left(W_{q-m}(K)\right) \\
F_{p}^{\prime \prime}\left(\mathscr{M}^{\prime \prime}\right)=O_{X} \otimes f^{-1}\left(W_{q-m}(\mathscr{M}) \cap F_{p}(\mathscr{M})\right) .
\end{gathered}
$$

(m9) Let $f: X \rightarrow Y$ be a projective morphism. For an object $C V=$ $(\mathcal{M}, F, K, W)$ of $\operatorname{MHM}(X)$ with $\mathrm{Gr}_{i}{ }^{W} C V=0$ for $i \neq k$, we have $\mathrm{Gr}_{i}{ }^{W}$ $\left(\mathcal{H}^{j} f_{!}(C)\right)=0$ for $i \neq j+k$ and $\mathrm{Gr}_{j+k}^{W}\left(\mathcal{H}^{j} f_{!}(C)\right)$ coincides with $\mathscr{H}^{j} f_{*}(\mathcal{M}, F$, $K)$ in the sence of Section 1.3.

(m10) For $C=(\mathcal{M}, F, K, W) \in M H M(X)$ and $C V^{\prime}=\left(\mathcal{M}^{\prime}, F^{\prime}, K^{\prime}, W^{\prime}\right) \in$ $\operatorname{MHM}\left(X^{\prime}\right)$ we have :

$$
\mathcal{C} \bowtie \mathcal{V}^{\prime}=\left(\mathscr{M} \bowtie \mathcal{M}^{\prime}, F^{\prime \prime}, K 凶 K^{\prime}, W^{\prime \prime}\right) \in M H M\left(X \times X^{\prime}\right),
$$

with $F_{p}^{\prime}=\sum_{q} F_{q} \rrbracket F_{p-q}^{\prime}$ and $W_{p}^{\prime \prime}=\sum_{q} W_{q} \rrbracket W_{p-q}^{\prime}$.

(m11) Let $f: \mathrm{X} \rightarrow \mathrm{Y}$ be a morphism of non-singular varieties and $T$ a non-singular variety. For a natural morphism $f \times 1: X \times T \rightarrow Y \times T$ we have :

$$
\begin{aligned}
& \mathscr{H}^{j}(f \times 1)_{!}\left(\mathcal{V} \mathcal{V}_{1} \rrbracket \mathcal{V}_{2}\right)=\left(\mathscr{H}^{j} f_{1} \mathcal{V}_{1}\right) \rrbracket \mathcal{V}_{2}, \\
& \mathscr{H}^{j}(f \times 1) *\left(C V_{1} \rrbracket C V_{2}\right)=\left(\mathcal{H}^{j} f^{*} C V_{1}\right) \bowtie C V_{2} .
\end{aligned}
$$

(m12) For a closed immersion $f: X \rightarrow Y, f_{1}\left(=\mathscr{H}^{0} f_{!}\right)$gives a category equivalence between $M H M(X)$ and the full subcategory of $M H M(Y)$ whose objects are supported in $X$. Its quasi-inverse is $\mathscr{H}^{0} f^{*}$.

(m13) Let $i: Y \rightarrow X$ be a closed immersion of non-singular varieties with $\operatorname{codim} Y=1$. Set $j: U=X-Y \hookrightarrow X$. For $C \mathcal{V} \in M H M(X)$ we have an exact sequence :

$$
0 \rightarrow i_{1}\left(\mathscr{K}^{-1} i^{*} C V\right) \rightarrow\left(\mathscr{H}^{0} j_{!}\right)\left(j^{*} C \mathcal{V}\right) \rightarrow C V \rightarrow i_{!}\left(\mathscr{K}^{0} i^{*} C V\right) \rightarrow 0 .
$$

(Note that $\mathscr{H}^{k} i^{*}=0$ for $k \neq 0,-1$ and $\mathscr{H}^{k} j_{1}=0$ for $k \neq 0$.)

(m14) $\left(\mathcal{L}_{X}, W\right)$ with $\operatorname{Gr}_{k}{ }^{W}\left(\mathcal{L}_{X}\right)=0(k \neq \operatorname{dim} X)$ belongs to $\operatorname{MHM}(X)$. Hence $(\mathcal{L}(Z, X), W)$ with $\operatorname{Gr}_{k}{ }^{w}(\mathcal{L}(Z, X))=0(k \neq \operatorname{dim} Z)$ belongs to $\operatorname{MHM}(X) . \quad\left(\mathcal{L}_{X}, W\right)$ and $(\mathcal{L}(Z, X), W)$ will be denoted by $\mathcal{L}_{X}$ and $\mathcal{L}(Z$, $X)$ in the following.

(m15) If $f: X \rightarrow Y$ is a morphism of non-singular varieties and $\phi_{Y}(H)$ $\in M H M(Y)$ for $H \in \operatorname{VSHM}(Y)^{p}$, then $\mathcal{H}^{j} f^{*}\left(\phi_{Y}(H)\right)=0$ for $j \neq \operatorname{dim} X$ 
- $\operatorname{dim} Y$ and $\mathscr{H}^{\operatorname{dim} X-\operatorname{dim} Y} f^{*}\left(\phi_{Y}(H)\right)=\phi_{X}\left(f^{*}(H)\right)$. Especially we have $\mathcal{H}^{\operatorname{dim} X-\operatorname{dim} Y} f^{*}\left(\mathcal{L}_{Y}\right)=\mathcal{L}_{X}$.

(m16) Let $X^{\prime} \stackrel{g^{\prime}}{\longrightarrow} X \stackrel{f}{\longrightarrow} Y$ and $X^{\prime} \stackrel{f^{\prime}}{\longrightarrow} Y^{\prime} \stackrel{g}{\longrightarrow} Y$ be morphisms of non-singular varieties which form a cartesian diagram.

(a) Assume that $f$ is projective and $\mathcal{V} \in M H M(X)$. If $\mathscr{H}^{i} g^{\prime *}(\mathcal{C V})=0$ $(i \neq k)$ and $\left(\mathcal{H}^{i} g^{*}\right)\left(\mathcal{H}^{j} f_{1}\right)(\mathcal{C V})=0(i \neq k)$ for any $j$, then $\left(\mathcal{H}^{k} g^{*}\right)\left(\mathscr{H}^{j} f_{1}\right)(\mathcal{C V})=$ $\left(\mathscr{H}^{j} f_{!}^{\prime}\right)\left(\mathcal{H}^{k} g^{\prime *}\right)(\mathcal{C V})$ for any $j$.

(b) If $g$ is smooth with relative dimension $m$, then $\left(\mathscr{H}^{m} g^{*}\right)\left(\mathscr{H}^{j} f_{!}\right)=$ $\left(\mathcal{H}^{j} f_{1}^{\prime}\right)\left(\mathscr{K}^{m} g^{\prime *}\right)$.

(m17) Let $f: X \rightarrow Y$ and $g: Y \rightarrow Z$ be morphisms of non-singular varieties.

(a) If $\mathcal{Q V} \in M H M(X)$ satisfies $\left(\mathscr{H}^{i} f_{!}\right)(\mathcal{C V})=0$ for $i \neq k$, then $\mathscr{H}^{j}(g \circ f)_{!}(\mathcal{C V})=\left(\mathcal{H}^{j-k} g_{1}\right)\left(\mathscr{H}^{k} f_{1}\right)(C V)$.

(b) If $g$ is a closed immersion, $\mathscr{H}^{\rho}\left(g^{\circ} f\right)_{!}=g_{1} \circ\left(\mathcal{H}^{\top} f_{1}\right)$.

(m18) Let $f: X \rightarrow Y$ and $g: Y \rightarrow Z$ be morphisms of non-singular varieties.

(a) If $C V \in M H M(Z)$ satisfies $\left(\mathscr{H}^{2} g^{*}\right)(\mathcal{C V})=0$ for $i \neq k$, then $\mathscr{H}^{j}(g \circ f)^{*}(\mathcal{C V})$ $=\left(\mathcal{H}^{j-k} f^{*}\right)\left(\mathcal{H}^{k} g^{*}\right)(\mathcal{C V})$.

(b) If $f$ is smooth with relative dimension $m, \mathscr{H}^{\circ}\left(g^{\circ} f\right)^{*}=\left(\mathscr{H}^{m} f^{*}\right)$ $\left(\mathscr{H}^{j-m} g^{*}\right)$.

Using the terminology of the derived category the properties (m16 18) above can be formulated without assuming vanishing of cohomologies ([Sa 4]). Here we formulate them in a weaker form.

We denote the Grothendieck group of $M H M(X)$ by $K H(X)$. For a morphism $f: X \rightarrow Y$ of non-singular varieties, $\mathbb{Z}$-linear maps $f_{1}: K H(X) \rightarrow$ $K H(Y)$ and $f^{*}: K H(Y) \rightarrow K H(X)$ are defined by $f_{1}([\mathcal{V}])=\sum_{j}(-1)^{\jmath}$ $\left[\mathscr{H}^{j} f_{1}(\mathcal{C V})\right]$ and $f^{*}([\mathcal{C V}])=\sum_{j}(-1)^{j}\left[\mathcal{H}^{j} f^{*}(\mathcal{C V})\right]$.

(m16') If $g$ is smooth or $f$ is projective in the cartesian diagram of (m16), then the two maps $g^{*} \circ f_{1}$ and $f^{\prime}, \circ g^{\prime *}$ from $K H(X)$ to $K H\left(Y^{\prime}\right)$ coincide.

(m17') Let $f: X \rightarrow Y$ and $g: Y \rightarrow Z$ be morphisms of non-singular varieties. Then the two maps $g_{1} \circ f_{1}$ and $(g \circ f)$, from $K H(X)$ to $K H(Z)$ coincide.

(m18') Let $f: X \rightarrow Y$ and $g: Y \rightarrow Z$ be morphisms of non-singular varieties. Then the two maps $f^{*} \circ g^{*}$ and $(g \circ f)^{*}$ from $K H(Z)$ to $K H(X)$ 
coincide.

Let $p t$ be the algebraic variety consisting of a single point. Set $R=$ $K H(p t) . \quad R$ is endowed with a ring structure via the tensor product $区$ (commutative with unit $\left[\mathcal{L}_{p t}\right]$ ) and the Laurent polynomial ring $\mathbb{Z}\left[q, q^{-1}\right]$ is a subring of $R\left(q^{i} \leftrightarrow\left[\mathcal{L}_{p t}(-i)\right]\right)$. We have an $R$-module structure on $K H(X)$ via the tensor product $\bigotimes$ and $f^{*}$ and $f$ : are $R$-homomorphisms.

\subsection{Hodge modules with group actions}

Let $G$ be an algebraic group over $C$ acting on a non-singular algebraic variety $X$. Let $m: G \times G \rightarrow G$ and $\sigma: G \times X \rightarrow X$ be the product in $G$ and the action of $G$ on $X$, respectively.

Definition. A Hodge module on $X$ with $G$-action is a pair $(C V, \varphi)$, where $C V$ is an object of $\operatorname{MHM}(X)$ and $\varphi:\left(\mathcal{H}^{\mathrm{dim} G} \sigma^{*}\right)(C) \rightarrow\left(\mathcal{H}^{\mathrm{dim} G} p_{2}{ }^{*}\right)(C)$ is an isomorphism in $\operatorname{MHM}(G \times X)$ such that the two morphisms $\left(\left(\mathcal{H}^{\operatorname{dim} G} p_{23}{ }^{*}\right) \varphi\right) \circ\left(\mathcal{H}^{\operatorname{dim} G}\left(1_{G} \times \sigma\right)^{*} \varphi\right)$ and $\left(\mathscr{H}^{\operatorname{dim} G}\left(m \times 1_{X}\right)^{*} \varphi\right)$ from $\mathscr{H}^{2 \operatorname{dim} G}\left(\sigma^{\circ}\left(1_{G}\right.\right.$ $\times \sigma))^{*}(C)=\mathscr{H}^{2 \operatorname{dim} G}\left(\sigma^{\circ}\left(m \times 1_{X}\right)\right)^{*}(C)$ to $\mathscr{H}^{2 \operatorname{dim} G}\left(p_{2}^{\circ} p_{23}\right)^{*}(C)=\mathscr{H}^{2 \operatorname{dim} G}\left(p_{2}^{\circ}(m\right.$ $\left.\left.\times 1_{X}\right)\right)^{*}(C)$ in $M H M(G \times G \times X)$ coincide. Here $p_{2}: G \times X \rightarrow X$ and $p_{23}: G$ $\times G \times X \rightarrow G \times X$ are projections.

The above formalism is due to Mumford. We define a category $\operatorname{MHM}(X, G)$ as follows. An object is a Hodge module with $G$-action. A morphism from $(C, \varphi)$ to $\left(C^{\prime}, \varphi^{\prime}\right)$ is a morphism $u: C V \rightarrow C V^{\prime}$ in $\operatorname{MHM}(X)$ satisfying $\left(\left(\mathcal{H}^{\operatorname{dim} G} p_{2}{ }^{*}\right) u\right) \circ \varphi=\varphi^{\prime} \circ\left(\left(\mathcal{H}^{\operatorname{dim} G} \sigma^{*}\right) u\right)$. It is easily seen that $\operatorname{MHM}(X, G)$ is an abelian category. We denote the Grothendieck group of $\operatorname{MHM}(X, G)$ by $K H^{G}(X)$.

For a $G$-equivariant morphism $f: X \rightarrow Y$ of non-singular varieties we have additive functors :

$$
\mathcal{H}^{j} f_{!}: \operatorname{MHM}(X, G) \rightarrow \operatorname{MHM}(Y, G), \quad \mathscr{H}^{j} f^{*}: \operatorname{MHM}(Y, G) \rightarrow \operatorname{MHM}(X, G) \text {, }
$$

which induce $\mathbb{Z}$-linear maps :

$$
f_{1}: K H^{G}(X) \rightarrow K H^{G}(Y), \quad f^{*}: K H^{G}(Y) \rightarrow K H^{G}(X) .
$$

When $X_{i}(i=1,2)$ are non-singular $G_{i}$-varieties, we have a bi-exact functor :

$$
凶: \operatorname{MHM}\left(X_{1}, G_{1}\right) \times M H M\left(X_{2}, G_{2}\right) \rightarrow \operatorname{MHM}\left(X_{1} \times X_{2}, G_{1} \times G_{2}\right),
$$

which induces a $\mathbb{Z}$-linear map: 


$$
\text { 凶: } K H^{G_{1}}\left(X_{1}\right) \underset{Z}{\otimes} K H^{G_{2}}\left(X_{2}\right) \rightarrow K H^{G_{1} \times G_{2}}\left(X_{1} \times X_{2}\right) .
$$

Especially $K H^{G}(X)$ is an $R(=K H(p t))$-module. It is seen that $f_{1}$ and $f^{*}$ are $R$-homomorphisms and $\bigotimes$ is $R$-bilinear.

For a $\mathbb{Q}$-local system $S$ on a non-singular variety $X$ we define $H_{s}=(S$, $F, W) \in \operatorname{VSHM}(X)$ by :

$$
F^{p}=\left(\begin{array}{ll}
\mathcal{M}(S) & (p \leqq 0) \\
0 & (p>0)
\end{array} \text { and } \quad W_{p}=\left(\begin{array}{ll}
(S, F) & (p \geqq 0) \\
0 & (p<0)
\end{array}\right.\right.
$$

If the monodromy representation of $S$ factors through a finite group, then $H_{s} \in \operatorname{VSHM}(X)^{p}$.

For a homogeneous space $X$ of $G$ we denote by $\operatorname{Loc}(X, G)$ the category of 2 -local systems on $X$ with $G$-actions. This category is equivalent to the category of finite dimensional representations over $\mathbb{Q}$ of the finite group $G^{x} /\left(G^{x}\right)^{\circ}$, where $x$ is a point of $X, G^{x}$ is its stabilizer in $G$ and $\left(G^{x}\right)^{\circ}$ is the connected component of $G^{x}$ containing the identity. For $S \in \operatorname{Loc}(X, G)$, $H_{S}$ belongs to $\operatorname{VSHM}(X)^{p}$ and is naturally endowed with a $G$-action.

Lemma 1.1. Let $X$ be a homogeneous space of $G$. We assume that irreducible representations of $G^{x} /\left(G^{x}\right)^{\circ}$ over $\mathbb{Q}$ are absolutely irreducible for some (and hence for any) point $x$ of $X$.

(i) For $S \in \operatorname{Loc}(X, G) \phi_{X}\left(H_{s}\right)$ belongs to $M H M(X)$ and is naturally endowed with an action of $G$.

(ii) If $S$ and $H$ are simple objects of $\operatorname{Loc}(X, G)$ and $M H M(p t)$ respectively, then $\phi_{X}\left(H_{S}\right) \bigotimes H$ is a simple object of $M H M(X, G)$.

(iii) If $\mathcal{Q}$ is an object of $\operatorname{MHM}(X, G)$ such that $\mathrm{Gr}_{2}{ }^{W} C V=0$ for $i \neq k$, then $C V$ is a direct sum of the simple objects of the type given in (ii) with $\mathrm{Gr}_{2}{ }^{W} H=0(i \neq k-\operatorname{dim} X)$.

(iv) $K H^{c}(X)$ is a free $R$-module with basis $\left\{\phi_{X}\left(H_{S}\right) \mid S\right.$ is a simple object of $\operatorname{Loc}(X, G)\}$.

We prepare a lemma in order to prove Lemma 1.1.

Lemma 1.2. Let $X$ be a homogeneous space of $G$. Choose a point $x$ of $X$ and set $p t=\{x\}, i: p t \hookrightarrow X$ and $q: X \rightarrow p t$. We assume that $G^{x}$ is connected. Then $\mathscr{T}^{-\mathrm{d} m X} i^{*}$ gives an equivalence of abelian categories $\operatorname{MHM}(X, G)$ and $\operatorname{MHM}(p t)$. Its quasi inverse is given by $\mathcal{H}^{\mathrm{dim} x} q^{*}$.

Proof. It follows from (p6) that any object of $\operatorname{MHM}(X, G)$ lies in the 
image of $\Phi_{X}$. Let $\operatorname{VSHM}(X, G)^{p}$ be the category of objects of $\operatorname{VSHM}(X)^{p}$ with $G$-actions. We have exact fully faithful functors $F_{X}: M H M(X, G) \rightarrow$ $\operatorname{VSHM}(X, G)^{p}$ and $F_{p t}: M H M(p t) \rightarrow S H M^{p}$. Since $G^{x}$ is connected, it is easily seen that $i^{*}: \operatorname{VSHM}(X, G)^{p} \rightarrow S H M^{p}$ gives an equivalence of categories with quasi-inverse $q^{*}$. By $(\mathrm{m} 15)$ we see that $i^{*} \circ F_{X}=F_{p t} \circ\left(\mathscr{H}^{-\operatorname{dim} X} i^{*}\right)$ and $q^{*} \circ F_{p t}=F_{X} \circ\left(\mathcal{H}^{\operatorname{dim} X} q^{*}\right)$. Hence the lemma.

\section{Proof of Lemma 1.1.}

Let $f: X_{0}=G /\left(G^{x}\right)^{\circ} \rightarrow X=G / G^{x}$ be the natural map and $S_{0}$ the $\boldsymbol{Q}$-local system on $X$ with $G$-action corresponding to the regular representation of $G^{x} /\left(G^{x}\right)^{\circ}$.

(i) Since $\mathcal{L}_{X_{0}} \in \operatorname{MHM}\left(X_{0}, G\right)$, we have $\Phi_{X}\left(H_{s_{0}}\right)=\left(\mathscr{H}^{0} f_{!}\right)\left(\mathcal{L}_{X_{0}}\right) \in$ $\operatorname{MHM}(X, G)$. Since any representation (over $\mathbf{Q}$ ) of a finite group is a direct sum of irreducible representations and since any irreducible representation is a direct summand of the regular representation, $\Phi_{X}\left(H_{S}\right) \in$ $\operatorname{MHM}(X, G)$ for any $S \in \operatorname{Loc}(X, G)$ by $(\mathrm{m} 2)$, and (i) is proved.

(ii) Let $\operatorname{SHM}\left(G^{x} /\left(G^{x}\right)^{\circ}\right)^{p}$ be the category of polarizable mixed Hodge structures with $G^{x} /\left(G^{x}\right)^{\circ}$-actions. As in the proof of Lemma 1.2 we have fully faithful functors:

$$
\operatorname{MHM}(X, G) \rightarrow \operatorname{VSHM}(X, G)^{p} \rightarrow \operatorname{SHM}\left(G^{x} /\left(G^{x}\right)^{\circ}\right)^{p} .
$$

Hence it is enough to show that $H \otimes V$ is a simple object of $\operatorname{SHM}\left(G^{x} /\right.$ $\left.\left(G^{x}\right)^{\circ}\right)^{p}$ if $H$ is a simple object of $S H M^{p}$ and $V$ is an irreducible $G^{x} /\left(G^{x}\right)^{\circ}$ module over $Q$. This follows from our assumption on $G^{x} /\left(G^{x}\right)^{\circ}$.

(iii) By Lemma 1.2 there exists an object $H$ of $M H M(p t)$ such that $\left(\mathscr{H}^{0} f^{*}\right)(\mathcal{C V})=\mathcal{L}_{X_{0}} \rrbracket H$ with $\mathrm{Gr}_{i}{ }^{W} H=0(i \neq k-\operatorname{dim} X)$. Since $S H(n)^{p}$ is a semisimple category, $H$ is a direct sum of simple objects by $(\mathrm{m} 2)$. Therefore the assertion follows from the fact that $\mathcal{V}$ is a direct summand of $\left(\mathcal{H}^{0} f_{1}\right)\left(\mathcal{H}^{0} f^{*}\right)(C V)=\Phi_{X}\left(H_{s_{0}}\right) \bowtie H$.

(iv) This follows from (ii) and (iii).

Proposition 1.3. Let $X$ be a non-singular G-variety and $Y$ a G-orbit containing $x \in X$. Set $\partial Y=\bar{Y}-Y$ and $i: Y \hookrightarrow X-\partial Y$. We assume that any irreducible representation of $G^{x} /\left(G^{x}\right)^{\circ}$ over $\mathbb{Q}$ is absolutely irreducible. For simple objects $H$ and $S$ of $M H M(p t)$ and $\operatorname{Loc}(Y, G)$ respectively, there exists a unique simple object $\mathcal{C V}$ of $M H M(X, G)$ such thät $\mathcal{C V} \mid X-\partial Y=$ $i_{1}\left(\phi_{Y}\left(H_{s}\right) \bigotimes H\right)$.

Proof. Since $H$ is simple, we have $\mathrm{Gr}_{j}{ }^{W} H=0(j \neq k)$ for some $k$. If 
such $\mathcal{V}$ exists, the underlying object $\mathcal{V}_{1}$ of $\operatorname{MHM}(X)$ satisfies the following condition :

$(P) C V_{1} \mid X-\partial Y=i_{1}\left(\Phi_{Y}\left(H_{s}\right) \bigotimes H\right), \mathrm{Gr}_{j}{ }^{W}\left(C_{1}\right)=0(j \neq n=k+\operatorname{dim} Y)$ and $\mathrm{Gr}_{n}{ }^{W}\left(C V_{1}\right)$ is an object of $M H_{\bar{Y}}(X, n)^{p}$.

If there exists $C V_{1} \in M H M(X)$ satisfying $(P)$, the action of $G$ on $\Phi_{Y}\left(H_{S}\right)$ $\bigotimes H$ uniquely extends to that of $G$ on $C_{1}$ by (p7) and the resulting object of $\operatorname{MHM}(X, G)$ is simple by (m12). Hence it is enough to prove the existence of $C V_{1} \in M H M(X)$ satisfying $(P)$. This follows from the desingularization theorem of Hironaka and the arguments as in the proof of Lemma 1.1.

Notation. We denote $\mathcal{C}$ in Proposition 1.3 by $\mathcal{L}(\bar{Y}, X, S, H)$. Set $\mathcal{L}(\bar{Y}, X, S)=\mathcal{L}\left(\bar{Y}, X, S, \mathcal{L}_{p t}\right)$.

Lemma 1.4. We have $\mathcal{L}(\bar{Y}, X, S, H)=\mathcal{L}(\bar{Y}, X, S) \bigotimes H$.

Proof. It is easy to see that $\mathcal{L}(\bar{Y}, X, S) \rrbracket H$ satisfies the condition $(P)$ in the proof of Proposition 1.3.

Proposition 1.5. Let $X$ be a non-singular G-variety with finitely many orbits. We assume that irreducible representations of $G^{x} /\left(G^{x}\right)^{\circ}$ over $Q$ are absolutely irreducible for any point $x$ of $X$.

(i) If $Q V$ is an object of $M H M(X, G)$ such that $\mathrm{Gr}_{2}{ }^{W} C V=0$ for $i \neq k$, then $\mathcal{C}$ is a direct sum of the simple objects of the type $\mathcal{L}(\bar{Y}, X, S, H)$, where $Y$ is a G-orbit, $S$ and $H$ are simple objects of $\operatorname{Loc}(Y, G)$ and $M H M(p t)$ respectively with $\mathrm{Gr}_{2}{ }^{W} H=0(i \neq k-\operatorname{dim} Y)$.

(ii) $K H^{G}(X)$ is a free $R$-module with basis $\{[\mathcal{L}(\bar{Y}, X, S)] \mid(Y, S)\}$, where $(Y, S)$ is running through pairs of a G-orbit $Y$ and a simple object $S$ of $\operatorname{Loc}(Y, G)$.

Proof. (i) Since $\mathrm{Gr}_{k}{ }^{W}(C)$ is an object of $M H(X, k)^{p}$, we have a direct sum decomposition $\mathrm{Gr}_{k}{ }^{W}(\mathcal{C})=\oplus_{Y} \mathcal{C V}_{Y}\left(Y\right.$ is a $G$-orbit and $\mathcal{V}_{Y}$ is an object of $\left.M H_{\bar{Y}}(X, k)^{p}\right)$ in $M H(X, k)^{p}$. Then each $\mathcal{V V}_{Y}$ (with $\mathrm{Gr}_{J}{ }^{W} \mathcal{V}_{Y}=0$ for $j \neq k)$ is an object of $\operatorname{MHM}(X, G)$. Hence we may assume that $\mathrm{Gr}_{k}{ }^{W}(C V)$ belongs to $M H_{\bar{Y}}(X, k)^{p}$. Set $\partial Y=\bar{Y}-Y$ and $i: Y \hookrightarrow X-\partial Y$. By $(\mathrm{m} 12)$ there exists an object $\mathcal{V}_{1}$ of $\operatorname{MHM}(Y, G)$ such that $\mathcal{V} \mid X-\partial Y=$ $i_{1}\left(\mathcal{V}_{1}\right)$ and $\mathrm{Gr}_{i}{ }^{W}\left(\mathcal{V}_{1}\right)=0(i \neq k)$. Hence the assertion follows from Lemma 1.1, Proposition 1.3 and (p7).

(ii) This follows from (i) and Lemma 1.4. 


\section{§2. Equivariant $\mathbb{K}$-theory and Hodge Modules with Group Actions}

\subsection{Equivariant - theory (see [Th])}

Let $G$ be an algebraic group and $i: X \hookrightarrow Y$ a $G$-equivariant closed immersion of $G$-varieties (not necessarily irreducible nor non-singular). We denote the abelian category consisting of coherent $O_{Y}$-modules with $G$ actions supported in $X$ by $C^{G}(X, Y)$. Let $K^{G}(X, Y)$ be its Grothendieck group. When $M$ is a bounded complex of $O_{Y}$-modules with $G$-action so that each $\mathscr{F}^{i}(M)$ belongs to $C^{G}(X, Y)$, we set $[M]=\sum_{i}(-1)^{i}\left[\mathscr{A}^{i}(M)\right] \in$ $K^{G}(X, Y)$. Since the exact functor $i_{*}: C^{G}(\mathrm{X}, \mathrm{X}) \rightarrow C^{G}(X, Y)$ induces an isomorphism $i_{*}: K^{G}(X, X) \rightarrow K^{G}(X, Y), K^{G}(X, Y)$ does not depend on the choice of the ambient space $Y$. When we do not have to specify $Y$ we denote it by $K^{G}(X)$. The abelian group $R_{G}=K^{G}(p t)$ is endowed with a ring structure and $K^{G}(X)$ is an $R_{G}$-module via the tensor product $\left(R_{G}\right.$ is called the representation ring of $G$.).

Let $Y_{i}(i=1,2)$ be $G$-varieties, $X_{i} G$-stable closed subvarieties of $Y_{i}$ and $f: Y_{1} \rightarrow Y_{2}$ be a $G$-equivariant morphism. When $f\left(X_{1}\right)$ is contained in $X_{2}$ and $X_{1} \rightarrow X_{2}$ is proper, an $R_{G}$-linear map:

$$
f_{*}: K^{G}\left(X_{1}, Y_{1}\right) \rightarrow K^{G}\left(X_{2}, Y_{2}\right)
$$

is defined by $f_{*}([M])=\left[\mathbb{R} f_{*}(M)\right]$. When $f^{-1}\left(X_{2}\right)$ is contained in $X_{1}$ and $Y_{2}$ is non-singular, an $R_{G}$-linear map:

$$
f^{*}: K^{G}\left(X_{2}, Y_{2}\right) \rightarrow K^{G}\left(X_{1}, Y_{1}\right)
$$

is defined by $f^{*}([M])=\left[\mathbb{L} f^{*}(M)\right]$. Let $X_{i}(i=1,2,3)$ be $G$-stable closed subvarieties of a non-singular $G$-variety $Y$ so that $X_{1} \cap X_{2} \subset X_{3}$. Then

$$
\otimes: K^{G}\left(X_{1}, Y\right){\underset{R}{G}}_{K^{G}}\left(X_{2}, Y\right) \rightarrow K^{G}\left(X_{3}, Y\right)
$$

is defined by $\left[M_{1}\right] \otimes\left[M_{2}\right]=\left[M_{1} \underset{O_{Y}}{\otimes} M_{2}\right]$. Note that $f_{*}$ does not depend on the choice of the ambient space while $f^{*}$ and $\otimes$ do.

The following well-known facts will be used frequently later.

Lemma 2.1. (projection formula). Let $f: Y_{1} \rightarrow Y_{2}$ be a G-equivariant morphism of non-singular $G$-varieties. When $M_{i}(i=1,2)$ are coherent $O_{Y_{2}}$-modules with $G$-actions so that $\operatorname{Supp}\left(M_{1}\right) \rightarrow Y_{2}$ is proper, we have: 


$$
\mathbb{R} f_{*}\left(M_{1} \underset{O_{Y_{1}}}{\stackrel{L}{L}} \mathbb{L} f^{*}\left(M_{2}\right)\right)=\mathbb{R} f_{*}\left(M_{1}\right) \underset{O_{Y_{2}}}{\stackrel{L}{\otimes}} M_{2}
$$

Lemma 2.2. (smooth base change theorem). Let $f: Y_{1} \rightarrow Y_{2}$ and $g_{2}$ : $Y_{2}^{\prime} \rightarrow Y_{2}$ be G-equivariant morphisms of non-singular $G$-varieties. Set $Y_{1}^{\prime}=$ $Y_{1} \times Y_{Y_{2}} Y_{2}^{\prime}$ and let $g_{1}: Y_{1}^{\prime} \rightarrow Y_{1}$ and $f^{\prime}: Y_{1}^{\prime} \rightarrow Y_{2}^{\prime}$ be natural maps. We assume that $g_{2}$ is smooth. When $M$ is a coherent $C_{Y_{1}}$-module with G-action so that Supp $M \rightarrow Y_{2}$ is proper, we have :

$$
\mathbb{L} g_{2}{ }^{*} \circ \mathbb{R} f_{*}(M)=\mathbb{R} f_{*}^{\prime} \circ \mathbb{L} g_{1}^{*}(M)
$$

2.2. Coherent sheaves on the cotangernt bundles associated to fillered $\mathbb{D}$-modules

For a non-singular variety $X$ over $\mathbb{C}$, we denote the cotangent bundle by $p: T^{*} X \rightarrow X$. The $O_{X}$-algebra $\operatorname{Gr}^{F} D_{X}$ is naturally identified with $p_{*} O_{T^{*} X}$. For an object $(\mathcal{M}, F)$ of $M F_{r h}\left(D_{X}\right)$ we have a coherent $O_{T^{*} X}$. module :

$$
\operatorname{gr}(\mathcal{M}, F):=O_{T^{*} X} \underset{p^{-1} \mathrm{Gr}^{F} D_{X}}{\otimes} p^{-1}\left(\mathrm{Gr}^{F} \mathcal{M}\right)
$$

The group $\mathbb{C}^{*}$ acts on $T^{*} X$ by $z^{\circ}(x, \xi)=(x, z \xi)$ ( $x$ is a coordinate of $X$ and $\xi$ is a coordinate of fibres.). We have a natural $\mathbb{C}^{*}$-action on $\operatorname{gr}(\mathscr{M}, F)$ by :

$$
\begin{aligned}
& z^{\circ}\left(f(x, \xi) \otimes m_{\imath}\right)=f\left(x, z^{-1} \xi\right) \otimes z^{-\imath} m_{\imath} \\
& \quad\left(z \in \mathbb{C}^{*}, f(x, \xi) \in O_{T^{*} X}, \quad m_{\imath} \in \mathrm{Gr}_{\imath}{ }^{F} \mathcal{M}\right) .
\end{aligned}
$$

For a morphism $f: X \rightarrow Y$ of non-singular varieties, set $\Omega_{X / Y}=$ $\Omega_{X} \otimes_{O_{X}} f^{*}\left(\Omega_{Y}{ }^{-1}\right)$. Consider the following commutative diagram:

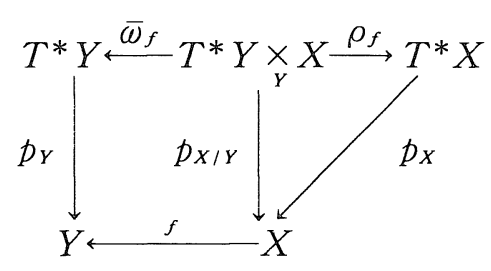

Here morphisms are the natural ones.

Lemma 2.3. Let $f: X \rightarrow Y$ be a projective morbhism of non-singular varieties and $(\mathcal{M}, F)$ an object of $M F_{r h}\left(D_{X}\right)$. If $f_{*}(\mathcal{M}, F)$ is strict, we have: 


$$
\operatorname{gr}\left(\mathscr{H}^{i} f_{*}(\mathcal{M}, F)\right)=\mathcal{H}^{i}\left(\boldsymbol{R} \bar{\omega}_{f *}\left(\boldsymbol{L} \rho_{f} *\left(\operatorname{gr}(\mathcal{M}, F) \underset{O_{r \cdot X}}{\otimes} p_{X}{ }^{*} \Omega_{X / Y}\right)\right)\right) \underset{C}{\otimes} V_{\operatorname{dim} X-\operatorname{dim} Y},
$$

as a coherent $O_{T^{*} X-m o d u l e}$ with $C^{*}$-action, where $V_{i}$ denotes the onedimensional $\mathbb{C}^{*}$-module such that the action of $z \in \mathbb{C}^{*}$ is given by the multiplication of $z^{i}$.

Proof. We first consider the case when $f$ is a projection. By the assumption $\mathscr{H}^{i}\left(\mathbb{R} f_{*}\left(F_{p}\left(D R_{X / Y}(\mathscr{M})\right)\right)\right) \rightarrow \mathscr{H}^{i}\left(\mathbb{R}_{*}\left(D R_{X / Y}(\mathscr{M})\right)\right)$ is injective for each $i$ and $p$ and we have $\mathscr{H}^{i} f_{*}(\mathscr{M}, F)=\left(\mathscr{H}{ }^{i}\left(\mathbb{R}_{*}\left(D R_{X / Y}(\mathscr{M})\right)\right), F\right)$ with $F_{p}\left(\mathscr{H}^{i}\left(\mathbb{R}_{*}\left(D R_{X / Y}(\mathscr{M})\right)\right)\right)=\mathscr{H}^{i}\left(\mathbb{R}_{*}\left(F_{p}\left(D R_{X / Y}(\mathscr{M})\right)\right)\right)$ (see Section 1.2). Apply $\boldsymbol{R}_{*}$ to the distinguished triangle:

$$
F_{p-1}\left(D R_{X / Y}(\mathscr{M})\right) \rightarrow F_{p}\left(D R_{X / Y}(\mathscr{M})\right) \rightarrow \operatorname{Gr}_{p}{ }^{F}\left(D R_{X / Y}(\mathscr{M})\right) \rightarrow F_{p-1}\left(D R_{X / Y}(\mathcal{M})\right)[1]
$$

and consider the long exact sequence of cohomologies. Then we have a short exact sequence :

$$
\begin{aligned}
& 0 \rightarrow \mathscr{H}^{i}\left(\boldsymbol{R} f_{*}\left(F_{p-1} D R_{X / Y}(\mathscr{M})\right)\right) \rightarrow \mathscr{H}^{i}\left(\boldsymbol{R} f_{*}\left(F_{p} D R_{X / Y}(\mathcal{M})\right)\right) \rightarrow \\
& \mathscr{H}^{i}\left(\boldsymbol{R} f_{*}\left(\operatorname{Gr}_{p}{ }^{F} D R_{X / Y}(\mathcal{M})\right) \rightarrow 0\right.
\end{aligned}
$$

for each $i$ and $p$. Hence $\operatorname{Gr}^{F}\left(\mathscr{H}^{i} f_{*}(\mathscr{M}, F)\right)=\mathscr{H}^{i}\left(\boldsymbol{R} f_{*}\left(\operatorname{Gr}^{F} D R_{X / Y}(\mathscr{M})\right)\right)$.

It is easily seen that the natural actions of $O_{X}$ and $f^{-1} \mathrm{Gr} D_{Y}$ on $\operatorname{Gr}^{F}\left(D R_{X / Y}(\mathcal{M})\right)$ induce an $f^{*} \operatorname{Gr} D_{Y}$-module structure on $\operatorname{Gr}^{F}\left(D R_{X / Y}(\mathcal{M})\right)$. By definition we have :

$$
\operatorname{Gr}^{F}\left(D R_{X / Y}(\mathcal{M})\right)=\operatorname{Gr}^{F} D_{Y-X} \underset{\operatorname{GrD} D_{X}}{\stackrel{L}{\otimes}} \operatorname{Gr}^{F^{\prime}} \mathcal{M}=f^{*} \operatorname{Gr} D_{Y} \underset{\operatorname{Gr} D_{X}}{\otimes} \operatorname{Gr}^{F^{\prime}} \mathcal{M} \underset{O_{X}}{\otimes} \Omega_{X / Y}^{L},
$$

where $F^{\prime}=F[\operatorname{dim} Y-\operatorname{dim} X]$. Set $V=T^{*} Y \underset{Y}{\times} X$ and $p=p_{X / Y}$ for simplicity. Then we have:

$$
\begin{aligned}
& \operatorname{gr}\left(\mathscr{H}^{i} f_{*}(\mathcal{M}, F)\right)
\end{aligned}
$$

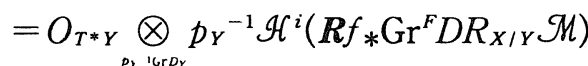

$$
\begin{aligned}
& =\mathscr{H}^{i}\left(O_{T^{*} Y} \otimes_{p_{1} \text {, lor } D_{Y}} p_{Y}{ }^{-1} \boldsymbol{R} f_{*} \mathrm{Gr}^{F} D R_{X / Y} \mathcal{M}\right) \\
& =\mathscr{H}^{i}\left(O_{T^{*} Y} \underset{\text { Prilcro, }}{\otimes} \boldsymbol{R} \bar{\omega}_{f * p^{-1}} \operatorname{Gr}^{F} D R_{X / Y} \mathscr{M}\right) \\
& =\mathscr{H}^{i}\left(\boldsymbol{R} \bar{\omega}_{f *}\left(O_{V_{p^{-1} f \cdot G r D_{Y}}}^{\otimes} p^{-1} \operatorname{Gr}^{F} D R_{X / Y} \mathscr{M}\right)\right.
\end{aligned}
$$




$$
\begin{aligned}
& =\mathscr{H}^{2}\left(\mathbb{R} \bar{\omega}_{f *}\left(O_{V} \bigotimes_{p^{\prime} \in \mathrm{C}_{1}}^{L} p^{-1}\left(\mathrm{Gr}^{F^{\prime}} \mathcal{M} \bigotimes_{O_{X}} \Omega_{X / Y}\right)\right)\right)
\end{aligned}
$$

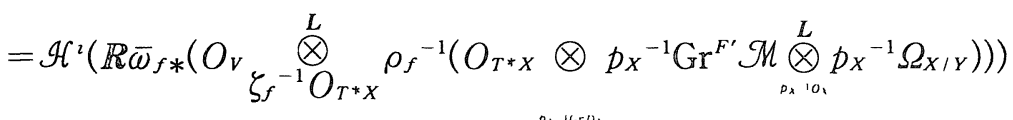

$$
\begin{aligned}
& =\mathscr{H}^{2}\left(\mathbb{R} \bar{\omega}_{f *} \mathbb{L} \rho_{f} *\left(\operatorname{gr}\left(\mathcal{M}, F^{\prime}\right) \underset{O_{l \cdot x}}{\bigotimes_{X}} p_{x}{ }^{*} \Omega_{X / Y}\right)\right) .
\end{aligned}
$$

Since $\operatorname{gr}(\mathcal{M}, F[i])=\operatorname{gr}(\mathcal{M}, F) \underset{C}{\otimes} V_{-\imath}$, the assertion is proved when $f$ is a projection. When $f$ is a closed immersion, our claim is shown by the similar arguments as above. Since any projective morphism is a composit of morphisms of these two types (a closed immersion followed by a projection), our assertion follows from the above two cases.

Remark. Saito informed us that the above Lemma follows directly from [Sa2 ; Section 2.3].

2.3. For a non-singular $G$-variety $X$ with finitely many $G$-orbits let $\Lambda$ $\left(=\Lambda_{(X, G)}\right)$ be the union of the conormal bundles $T_{o}^{*} X$ of $G$-orbits $O$. It is a $G \times \mathbb{C}^{*}$-stable closed subvariety of $T^{*} X$. For an object $C V=(\mathcal{M}, F, K$, $W)$ of $\operatorname{MHM}(X, G)$ we have an object $\operatorname{gr} C V:=\operatorname{gr}(\mathcal{M}, F)$ of $C^{G \times C^{*}}\left(\Lambda, T^{*} X\right)$. This induces a $\mathbb{Z}$-linear map:

$$
\text { gr : } K H^{G}(X) \rightarrow K^{G \times C^{*}}(\Lambda)=K^{G \times C^{*}}\left(\Lambda, T^{*} X\right) \text {. }
$$

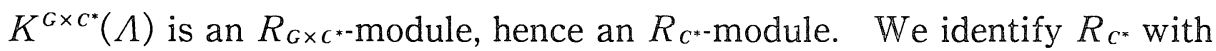
$\mathbb{Z}\left[q, q^{-1}\right]$ via $\left[V_{\imath}\right] \leftrightarrow q^{2}$. On the other hand $K H^{G}(X)$ is an $R(=K H(p t))$. module, hence a $\mathbb{Z}\left[q, q^{-1}\right]$-module (see Section 1.3 ). It is easily seen from the definition that $\mathrm{gr}$ is a homomorphism of $\mathbb{Z}\left[q, q^{-1}\right]$-module. The following lemma is clear from Lemma 2.3 (compare with [La]).

Lemma 2.4. Let $f: X \rightarrow Y$ be a projective G-equivariant morphism of non-singular $G$-varieties with finitely many G-orbits. Then for $u \in$ $K H^{G}(X)$ we have :

$$
\operatorname{gr}(f, u)=q^{\operatorname{dim} X-\operatorname{dim} Y} \bar{\omega}_{f *}\left(\rho_{f}^{*}\left(\operatorname{gr}(u) \otimes p_{X} *\left[\Omega_{X / Y}\right]\right)\right) .
$$

The following is also clear from the definition.

Lemma 2.5. Let $f: X \rightarrow Y$ be a smooth G-equivariant morphism of non-singular $G$-varieties with finitely many G-orbits. Then for $u \in$ $K H^{G}(Y)$ we have : 


$$
\operatorname{gr}\left(f^{*} u\right)=(-1)^{\operatorname{dim} X-\operatorname{dim} Y} \rho_{f *}\left(\bar{\omega}_{f}^{*}(\operatorname{gr}(u))\right)
$$

\section{$\S 3$. Realization of Hecke Algebras of Weyl Groups}

In Sections 3 to $5 G$ is a connected reductive algebraic group over $\mathbb{C}$ and $X$ is the flag variety of $G$.

3.1. It is well-known that the set of $G$-orbits on $X \times X$ is parametrized by the Weyl group $W$. In fact if we identify $X$ with the quotient $G / B$ for a fixed Borel subgroup $B, X \times X$ is the disjoint union of the $G$-orbits $Y_{w}$ containing $(B, w B)$, where $w$ is running through the elements of $W$. Moreover we have $\operatorname{dim} Y_{w}=N+l(w)$ and $\bar{Y}_{w} \supset \bar{Y}_{y}$ if and only if $w \geqq y$, where $N=\operatorname{dim} X, l(w)$ is the length of $w$ and $\geqq$ is the Bruhat ordering on $W$. These facts are direct consequences of the corresponding facts concerning $B$-orbits on $X$.

Let $i_{w}: Y_{w} \rightarrow X \times X$ be the natural inclusion. We set:

$$
\mathcal{L}_{w}=\mathcal{L}\left(\bar{Y}_{w}, X \times X\right) \text { and } \mathcal{M}_{w}=\mathscr{H}^{o} i_{w !}\left(\mathcal{L}_{Y_{w}}\right) \text {. }
$$

They are objects of $M H M(X \times X, G)$. Note that $\mathcal{H}^{j} i_{w !}\left(\mathcal{L}_{Y_{w}}\right)=0$ for $j \neq 0$ since $i_{w}$ is an affine morphism. By Proposition 1.5 the $R$-module $K H^{G}(X$ $\times X)$ has a free basis $\left\{\left[\mathcal{L}_{w}\right] \mid w \in W\right\}$. Since $\left[\mathcal{M}_{w}\right]$ belongs to $\left[\mathcal{L}_{w}\right]$ $+\sum_{y<w} R\left[\mathcal{L}_{y}\right],\left\{\left[\mathscr{M}_{w}\right] \mid w \in W\right\}$ is also a free basis of $K H^{G}(X \times X)$.

We define $p_{13}: X \times X \times X \rightarrow X \times X$ and $r: X \times X \times X \rightarrow X \times X \times X \times X$ by $p_{13}(a, b, c)=(a, c)$ and $r(a, b, c)=(a, b, b, c)$. For $u, v \in K H^{c}(X \times X)$ set

$$
u \cdot v=(-1)^{N} p_{13 !} r^{*}(u \bowtie v) \in K H^{G}(X \times X) .
$$

It follows from $\left(\mathrm{m} 16^{\prime}\right)$ that this product satisfies the associativity. For $s \in$ $S=\{$ simple reflection of $W\}$ let $X^{s}$ be the generalized flag variety consisting of parabolic subgroups with semisimple rank 1 corresponding to $s$ and $\pi_{s}: X \rightarrow X^{s}$ the natural morphism.

Lemma 3.1. For $u \in K H^{G}(X \times X), s \in S$ and $w \in W$ we have :

(i) $\left[\mathcal{L}_{e}\right] \cdot u=u \cdot\left[\mathcal{L}_{e}\right]=u$,

(ii) $\left[\mathcal{L}_{s}\right] \cdot u=-\left(\pi_{s} \times 1\right)^{*}\left(\pi_{s} \times 1\right)_{!}(u)$,

(ii') $u \cdot\left[\mathcal{L}_{s}\right]=-\left(1 \times \pi_{s}\right) *\left(1 \times \pi_{s}\right)_{!}(u)$,

(iii) $\left[\mathscr{M}_{s}\right]=\left[\mathcal{L}_{s}\right]+\left[\mathcal{L}_{e}\right]$ and $\left[\mathcal{L}_{s}\right]=\left[\mathscr{M}_{s}\right]-\left[\mathcal{M}_{e}\right]$, 


$$
\begin{aligned}
& \left(\operatorname{iv} \mathcal{L} \mathcal{L}_{s}\right] \cdot\left[\mathcal{L}_{s}\right]=-(q+1)\left[\mathcal{L}_{s}\right], \\
& (\operatorname{v})\left[\mathscr{M}_{s}\right] \cdot\left[\mathscr{M}_{w}\right]=\left[\mathscr{M}_{s w}\right] \text { if } \quad s w>w, \\
& \left(\operatorname{viD} \mathcal{M}_{w}\right] \cdot\left[\mathscr{M}_{s}\right]=\left[\mathscr{M}_{w s}\right] \text { if } \quad w s>w .
\end{aligned}
$$

Proof. First note that $\bar{Y}_{s}$ is non-singular and hence $\mathcal{L}_{s}=\bar{i}_{s^{\prime}}\left(\mathcal{L}_{\bar{Y}^{s}}\right)$ with $\bar{i}_{s}: \bar{Y}_{s} \hookrightarrow X \times X$. Thus (i), (ii), (ii'), (v) ( $\left.\mathrm{v}^{\prime}\right)$ follow from (m16'), and (iii) follows from (m13). (iv) is a consequence of (ii), (m16') and Example in 1.3 .

We see from Lemma 1.1 that $K H^{G}\left(Y_{w}\right)$ is a free $R$-module of rank one generated by $\left[\mathcal{L}_{Y_{w}}\right]$. We define an $R$-linear map:

$$
h: K H^{G}(X \times X) \rightarrow R \underset{Z_{\left[q, q^{-1}\right]}}{\otimes} H(W)
$$

by :

$$
h(u)=\sum_{w \in W}(-1)^{l}\left({ }^{w)} h_{w}(u) T_{w} \quad \text { with } \quad i_{w} *(u)=h_{w}(u)\left[\mathcal{L}_{Y_{w}}\right] .\right.
$$

Proposition 3.2. $h$ is an isomorphism of $R$-algebras.

Proof. We see easily from (m3), (m4), (m12) that $h\left(\left[\mathcal{M}_{w}\right]\right)=$ $(-1)^{l}{ }^{w)} T_{w}$. Hence the assertion follows from Lemma 3.1.

Lemma 3.3. Let $C V_{1}$ and $C V_{2}$ be objects of $M H M(X \times X, G)$.

(i) $\mathscr{H}^{\jmath} r^{*}\left(V_{1} 区 \subset V_{2}\right)=0$ for $j \neq-N$.

$$
\text { Hence }\left[C V_{1}\right] \cdot\left[C V_{2}\right]=\sum_{j}(-1)^{\jmath}\left[\left(\mathscr{H}^{{ }^{j} p_{13}}\right)\left(\mathscr{H}^{-N} r^{*}\right)\left(C V_{1} \unrhd C V_{2}\right)\right] \text {. }
$$

(ii) $\quad W_{p}\left(\mathscr{H}^{-N} r^{*}\left(C V_{1} \rrbracket C V_{2}\right)\right)=\left(\mathcal{H}^{-N} r^{*}\right)\left(\sum_{q} W_{q}\left(C V_{1}\right) \rrbracket w_{p-q+N}\left(C V_{2}\right)\right)$.

Proof. Fix $x_{0} \in X$ and let $U$ be the unipotent radical of a Borel subgroup which is opposite to the Borel subgroup corresponding to $x_{0}$. We define

$$
\begin{aligned}
& \varphi_{1}: X \times U \rightarrow X \times X, \quad \varphi_{2}: U \times X \rightarrow X \times X, \quad \psi: X \times U \times X \rightarrow X \times X \times X, \\
& k_{1}: X \rightarrow X \times X, \quad k_{2}: X \rightarrow X \times X
\end{aligned}
$$

by

$$
\begin{aligned}
& \varphi_{1}(x, u)=\left(u \cdot x, u \cdot x_{0}\right), \quad \varphi_{2}(u, y)=\left(u \cdot x_{0}, u \cdot y\right), \\
& \psi(x, u, y)=\left(u \cdot x, u \cdot x_{0}, u \cdot y\right) \\
& k_{1}(x)=\left(x, x_{0}\right), \quad k_{2}(y)=\left(x_{0}, y\right) .
\end{aligned}
$$

$\varphi_{1}, \varphi_{2}, \psi$ are open immersions and $k_{1}, k_{2}$ are closed immersions. Consider 
the commutative diagram :

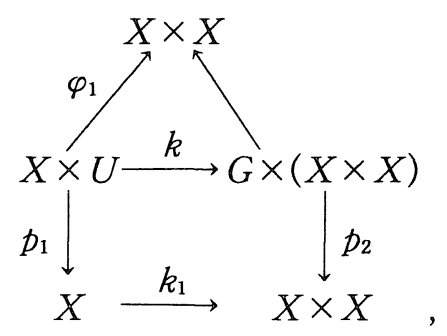

where $k(x, u)=\left(u, x, x_{0}\right)$ and $\sigma(g, x, y)=(g \circ x, g \circ y)$. For an object $Q$ of $\operatorname{MHM}(X \times X, G)$ we have :

$$
\begin{aligned}
& \mathscr{H}^{i} \varphi_{1}{ }^{*}(C)=\left(\mathcal{H}^{i-\operatorname{dim} G} k^{*}\right)\left(\mathcal{H}^{\operatorname{dim} G} \sigma^{*}\right)(C) \\
& \simeq\left(\mathscr{H}^{i-\operatorname{dim} G} k^{*}\right)\left(\mathcal{H}^{\operatorname{dim} G} p_{2}{ }^{*}\right)(\mathcal{C V}) \\
& =\mathscr{H}^{i}\left(p_{2} \circ k\right) *(\mathcal{V}) \\
& =\left(\mathscr{H}^{N} p_{1}{ }^{*}\right)\left(\mathscr{H}^{i-N} k_{1}{ }^{*}\right)(\mathcal{C}) \\
& =\left(\mathscr{K}^{i-N} k_{1}^{*}\right)(C) \times \mathcal{L}_{U}
\end{aligned}
$$

in $M H M(X \times U)$. Since $\varphi_{1}$ is an open immersion, $\mathscr{H}^{2} \varphi_{1} *(\mathcal{V})=0$ for $i \neq 0$. Hence $\mathscr{H}^{i} k_{1}{ }^{*}(\mathcal{C})=0$ for $i \neq-N, \mathscr{H}^{-N} k_{1}{ }^{*}$ is an exact functor from $M H M(X$ $\times X, G)$ to $\operatorname{MHM}(X)$ and $\varphi_{1}{ }^{*}(\mathcal{V}) \simeq\left(\mathcal{H}^{-N} k_{1}{ }^{*}(C)\right) \triangle \mathcal{L}_{U}$. Under this identification we have:

$$
\begin{aligned}
& \left(\mathcal{H}^{-N} k_{1}{ }^{*}\right)\left(W_{p}(C)\right) \bowtie \mathcal{L}_{U} \simeq \varphi_{1}{ }^{*}\left(W_{p}(\mathcal{V})\right) \\
& =W_{p}\left(\varphi_{1}^{*}(\mathcal{C})\right) \\
& \left.\simeq W_{p}\left(\left(\mathcal{H}^{-N} k_{1} *(C)\right)\right) 区 \mathcal{L}_{U}\right) \\
& =W_{p-N}\left(\mathcal{K}^{-N} k_{1} *(C)\right) \bowtie \mathcal{L}_{U},
\end{aligned}
$$

and hence $\left.W_{p}\left(\mathscr{H}^{-N} k_{1} *(C)\right)\right)=\mathscr{H}^{-N} k_{1}{ }^{*}\left(W_{p+N}(C)\right)$. In consequence we have :

$$
\begin{aligned}
& \varphi_{1}^{*}\left(\mathcal{V}_{1}\right) \simeq\left(\mathcal{H}^{-N} k_{1} *\left(\mathcal{V}_{1}\right)\right) 区 \mathcal{L}_{U}, \\
& W_{p}\left(\mathcal{H}^{-N} k_{1} *\left(\mathcal{V}_{1}\right)\right)=\mathcal{H}^{-N} k_{1} *\left(W_{p+N}\left(C V_{1}\right)\right) .
\end{aligned}
$$

Similarly we have :

$$
\begin{aligned}
& \varphi_{2}{ }^{*}\left(C V_{2}\right) \simeq \mathcal{L}_{U} \square\left(\mathcal{H}^{-N} k_{2} *\left(C V_{2}\right)\right), \\
& W_{p}\left(\mathcal{H}^{-N} k_{2} *\left(\mathcal{V}_{2}\right)\right)=\mathcal{H}^{-N} k_{2} *\left(W_{p+N}\left(C_{2}\right)\right) .
\end{aligned}
$$

Let $\Delta: U \rightarrow U \times U$ be the diagonal embedding. Since $r \circ \psi=\left(\varphi_{1} \times \varphi_{2}\right) \circ(1 \times \Delta$ 
$\times 1$ ), it is easily seen that:

$$
\begin{aligned}
& \psi^{*}\left(\mathcal{H}^{i} r^{*}\left(\mathcal{V}_{1} \rrbracket \subset \mathcal{V}_{2}\right)\right)=0 \text { for } i \neq-N,
\end{aligned}
$$

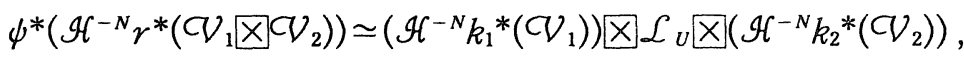

$$
\begin{aligned}
& \phi^{*}\left(\mathcal{H}^{-N} \gamma^{*}\left(W_{p}\left(\mathcal{V}_{1} \rrbracket \mathcal{V}_{2}\right)\right)\right)=\psi^{*}\left(\mathcal{H}^{-N} \gamma^{*}\right)\left(\sum_{q} W_{q}\left(\mathcal{V}_{1}\right) \rrbracket W_{p-q+N}\left(\mathcal{V}_{2}\right)\right) .
\end{aligned}
$$

Hence the lemma.

\subsection{Kazhdan-Lusztig Polynomials and $\mathcal{L}_{w}$}

For each $w \in W$ there exists a unique element $C_{w}{ }^{\prime \prime}$ of $H(W)$ of the form :

$$
C_{w}{ }^{\prime \prime}=(-1)^{l(w)} \sum_{y \leqq w} P_{y, w}(q) T_{y}=(-q)^{l(w)} \sum_{y \leqq w} P_{y, w}\left(q^{-1}\right) T_{y^{-1}}^{-1}
$$

such that $P_{w, w}(q)=1$ and $P_{y, w}(q)$ is a polynomial in $q$ with degree $\leqq(l(w)$ $-l(y)-1) / 2$ for $y<w([\mathrm{KL} 1])$. For $y<w$ with $l(w)-l(y)$ odd, we denote the coefficient of $q^{(l(w)-l(y)-1) / 2}$ in $P_{y, w}(q)$ by $\mu(y, w)$. The following two lemmas are known.

Lemma 3.4 ([KL1]). Let $s \in S$ and $w \in W$.

(i) If $s w>w$, we have:

$$
C_{s}{ }^{\prime \prime} C_{w}{ }^{\prime \prime}=C_{s w}{ }^{\prime \prime}+\sum_{z} \mu(z, w) q^{(l(w)-l(z)+1) / 2} C_{z}{ }^{\prime \prime},
$$

where $z$ is running through elements of $W$ so that $z<w$, sz<z and $l(w)$ $-l(z)$ is odd.

(ii) If $s w<w$, we have :

$$
C_{s}{ }^{\prime \prime} C_{w}{ }^{\prime \prime}=-(q+1) C_{w}^{\prime \prime} .
$$

Lemma 3.5 ([KL2], [Sp]). Let $s \in S$ and $w, y \in W$.

(i) If $j+l(w)-l(y)$ is odd, then ${ }^{p} \mathscr{H}^{j} i_{y}{ }^{*}\left(\mathscr{K}\left(\bar{Y}_{w}\right)\right)=0$.

(ii) If $s w>w$, we have:

$$
\begin{aligned}
& { }^{p} \mathcal{K}^{1}\left(\pi_{s} \times 1\right)^{* p} \mathscr{K}^{j}\left(\pi_{s} \times 1\right),\left(\mathscr{K}\left(\bar{Y}_{w}\right)\right) \\
& =\left(\begin{array}{ll}
0 & (j \neq 0) \\
\mathscr{C}\left(\bar{Y}_{s w}\right) \oplus\left(\oplus_{z} \mathscr{C}\left(\bar{Y}_{z}\right) \oplus \mu(z, w)\right. & (j=0),
\end{array}\right.
\end{aligned}
$$

where $z$ is running through elements of $W$ so that $z<w, s z<z$ and $l(w)$ $-l(z)$ is odd. 
(iii) If $s w<w$, we have:

$$
{ }^{p} \mathscr{K}^{1}\left(\pi_{s} \times 1\right)^{* p} \mathscr{H}^{j}\left(\pi_{s} \times 1\right)_{!}\left(\mathscr{C}\left(\bar{Y}_{w}\right)\right)=\left(\begin{array}{ll}
0 & (j \neq \pm 1) \\
\mathscr{C}\left(\bar{Y}_{w}\right) & (j= \pm 1) .
\end{array}\right.
$$

Lemma 3.6. Assume $s w>w$ for $s \in S$ and $w \in W$.

(i) $\mathscr{H}^{1}\left(\pi_{s} \times 1\right)^{*} \mathscr{H}^{j}\left(\pi_{s} \times 1\right)_{!}\left(\mathcal{L}_{w}\right)=0$ for $j \neq 0$.

(ii) $\operatorname{Gr}_{k}{ }^{W}\left(\mathcal{H}^{1}\left(\pi_{s} \times 1\right) * \mathcal{H}^{0}\left(\pi_{s} \times 1\right)_{1}\left(\mathcal{L}_{w}\right)\right)=0$ for $k \neq N+l(w)+1$.

(iii) $\mathcal{L}_{s w}$ is a direct summand of $\mathscr{H}^{1}\left(\pi_{s} \times 1\right)^{*} \mathscr{H}^{0}\left(\pi_{s} \times 1\right)_{!}\left(\mathcal{L}_{w}\right)$.

Proof. (i) is clear from Lemma 3.5. (ii) follows from the fact that $\pi_{s} \times 1$ is projective and smooth with relative dimension 1 .

(iii) It follows from (m16) that $\mathcal{L}_{s w}$ and $\mathscr{H}^{1}\left(\pi_{s} \times 1\right) * \mathcal{H}^{0}\left(\pi_{s} \times 1\right)_{!}\left(\mathcal{L}_{w}\right)$ coincide on $(X \times X)-\left(\bar{Y}_{s w}-Y_{s w}\right)$. Hence the assertion follows from (ii).

For a non-singular $G$-variety $V$ we denote by $K H^{G}(V)^{+}$(resp. $R^{+}$) the set of the elements in $K H^{G}(V)$ (resp. $R$ ) represented by objects of $M H M(V$, $G)$ (resp. $M H M(p t)$ ). Let $\left\{H_{\gamma}(i) \mid \gamma \in \Gamma, i \in \mathbb{Z}\right\}$ be the set of isomorphism classes of simple objects of $M H M(p t)$. For each $\gamma \in \Gamma$ an integer $n_{\gamma}$ is determined by $\mathrm{Gr}_{2}{ }^{W}\left(H_{\gamma}\right)=0$ for $i \neq n_{\gamma}$. We may assume that $H_{\gamma_{0}}=\mathcal{L}_{p t}$. Then we have:

$$
\begin{aligned}
& R^{+}=\underset{\substack{\gamma \in \Gamma \\
i \in Z}}{\bigoplus} \mathbb{Z}_{\geq 0}\left[H_{\gamma}(i)\right]=\bigoplus_{\gamma \in \Gamma} \mathbb{Z}_{\geq 0}\left[q, q^{-1}\right]\left[H_{\gamma}\right] \\
& K H^{G}(X \times X)^{+}=\bigoplus_{w \in W} R^{+}\left[\mathcal{L}_{w}\right] \\
& K H^{G}\left(Y_{y}\right)^{+}=R^{+}\left[\mathcal{L}_{Y_{y}}\right] .
\end{aligned}
$$

Proposition 3.7. Let $s \in S$ and $y, w \in W$.

(i) $h_{y}\left(\left[\mathcal{L}_{w}\right]\right) \in(-1)^{l(w)-l(y)} \mathbb{Z}_{\geq 0}\left[q, q^{-1}\right]$.

(ii) If $s w>w$, we have:

$$
\begin{aligned}
& \mathcal{H}^{1}\left(\pi_{s} \times 1\right)^{*} \mathcal{H}^{0}\left(\pi_{s} \times 1\right)_{1}\left(\mathcal{L}_{w}\right) \\
& =\mathcal{L}_{s w} \oplus\left(\oplus_{z} \mathcal{L}_{z}(-(l(w)-l(z)+1) / 2)^{\oplus \mu(z, w)}\right),
\end{aligned}
$$

where $z$ is running through elements of $W$ so that $z<w, s z<z$ and $l(w)$ $-l(z)$ is odd.

(iii) If $s w<w$, we have: 


$$
\mathscr{H}^{1}\left(\pi_{s} \times 1\right)^{*} \mathcal{H}^{j}\left(\pi_{s} \times 1\right):\left(\mathcal{L}_{w}\right)=\left(\begin{array}{ll}
\mathcal{L}_{w}(1) & (j=-1) \\
\mathcal{L}_{w} & (j=1) \\
0 & (j \neq \pm 1)
\end{array}\right.
$$

(iv) $h\left(\left[\mathcal{L}_{w}\right]\right)=C_{w}{ }^{\prime \prime}$.

Proof. We first prove (i) and (ii). Assume $s w>w$ for $w \in W$ and $s \in$ S. By induction we have only to show the statement:

(*) $\quad h_{y}\left(\left[\mathcal{L}_{s w}\right]\right) \in(-1)^{l(w)-l(y)+1} \mathbb{Z}_{\geqq 0}\left[q, q^{-1}\right]$ for any $y \in W$ and $\mathcal{H}^{1}\left(\pi_{s} \times 1\right)^{*} \mathcal{H}^{0}\left(\pi_{s} \times 1\right),\left(\mathcal{L}_{w}\right)=\mathcal{L}_{s w} \oplus\left(\underset{z}{\oplus} \mathcal{L}_{z}(-(l(w)-l(z)+1) / 2)^{\oplus(z, w)}\right)$, where $z$ is running through elements of $W$ so that $z<w, s z<z$ and $l(w)$ $-l(z)$ is odd,

assuming :

(* * * $) \quad h_{y}\left(\left[\mathcal{L}_{z}\right]\right) \in(-1)^{l(z)-l(y)} \mathbb{Z}_{\geq 0}\left[q, q^{-1}\right]$ for any $y, z \in W$ with $l(z) \leqq$ $l(w)$.

Set $\mathcal{V}=\mathscr{H}^{1}\left(\pi_{s} \times 1\right) * \mathcal{H}^{0}\left(\pi_{s} \times 1\right),\left(\mathcal{L}_{w}\right)$. It follows from Lemma 3.6 (ii), (iii) that we have $\mathcal{Q}=\mathcal{L}_{s w} \oplus\left(\underset{(z, r, i) \in J}{\oplus_{\gamma}}\left(H_{\gamma}(i)^{\oplus m_{z, \gamma, z}}\right) \bowtie \mathcal{L}_{z}\right)$ for some integers

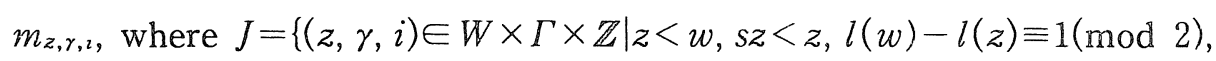
$\left.n_{\gamma}=l(w)-l(z)+2 i+1\right\}$. Since $h_{y}\left(\left[\mathcal{L}_{s w}\right]\right)=(-1)^{l(w)-l(y)+1} \sum_{\gamma \in \Gamma} f_{\gamma}(q)\left[H_{\gamma}\right]$ for some $f_{\gamma}(q) \in \mathbb{Z}_{\geq 0}\left[q, q^{-1}\right]$ by Lemma 3.5(i), we have:

$$
(-1)^{l(w)-l(y)+1} h_{y}([C V])=\sum_{\gamma \in \Gamma} k_{\gamma}(q)\left[H_{\gamma}\right]
$$

with

$$
k_{\gamma}(q)=f_{\gamma}(q)+\sum_{(z, \gamma, i) \in J} m_{z, \gamma, i} q^{-i}\left((-1)^{l(z)-l(y)} h_{y}\left(\left[\mathcal{L}_{z}\right]\right)\right) .
$$

On the other hand we have $h([C \mathcal{V}])=h\left(\left[\mathcal{L}_{s}\right] \cdot\left[\mathcal{L}_{w}\right]\right)=h\left(\left[\mathcal{L}_{s}\right]\right) h\left(\left[\mathcal{L}_{w}\right]\right)$ $\in H(W)$ and hence $k_{\gamma}(q)=0$ for $\gamma \neq \gamma_{0}$. Since $f_{\gamma}(q),(-1)^{\iota(z)-l(y)} h_{y}\left(\left[\mathcal{L}_{z}\right]\right) \in$ $\mathbb{Z}_{\geqq 0}\left[q, q^{-1}\right]$ and $m_{z, \gamma, i} \in \mathbb{Z}_{\geqq 0}$, we have $f_{\gamma}(q)=0$ for $\gamma \neq \gamma_{0}$ and $m_{z, \gamma, 2}=0$ for $(z$, $\gamma, i) \in J$ with $\gamma \neq \gamma_{0}$. Thus our assertion follows from Lemma 3.5(ii) and Lemma 3.6(ii).

(iv) This is easily proved by induction on $l(w)$ in view of (ii) and Lemma 3.4(i).

(iii) Since $\operatorname{Gr}_{k}{ }^{W}\left(\mathcal{H}^{1}\left(\pi_{s} \times 1\right) * \mathcal{K}^{j}\left(\pi_{s} \times 1\right),\left(\mathcal{L}_{w}\right)\right)=0$ for $k \neq N+l(w)+j$ +1 , the assertion follows from (iv) and Lemma 3.5 (ii). 
Definition. Let $\mathcal{A}$ be the full subcategory of $M H M(X \times X, G)$ consisting of $C \mathcal{V} \in M H M(X \times X, G)$ so that $\mathrm{Gr}_{k}{ }^{W} C \mathcal{V}$ is a direct sum of the simple objects of the form $\mathcal{L}_{w}(n)$ with $l(w)+N-2 n=k$.

It is easily seen that $\mathcal{A}$ is an abelian category.

Lemma 3.8. For $\mathcal{V} \in M H M(X \times X, G), h([\mathcal{C V}]) \in H(W)$ if and only if $\mathscr{V} \in \mathcal{A}$. Especially, we have $\mathscr{M}_{w} \in \mathcal{A}$ for any $w \in W$.

Proof. If $[\mathcal{C V}]=\sum_{\substack{\gamma \in T \\ w \in W}} f_{\gamma, w}(q)\left[H_{r} \bigotimes \mathcal{L}_{w}\right] \quad\left(f_{\gamma, w}(q) \in Z_{\geqq 0}\left[q, q^{-1}\right]\right) \quad$ for $\quad$ an object $\mathcal{V}$ of $M H M(X \times X, G)$, then $h([\mathcal{C}])=\sum_{\substack{\gamma \in \Gamma \\ w \in W}} f_{r, w}(q)\left[H_{r}\right] C_{w}{ }^{\prime \prime}$. Thus $h([\mathcal{V}]) \in H(W)$ if and only if composition factors of $\mathcal{V}$ are of the form $\mathcal{L}$ ${ }_{w}(n)$ with $w \in W$ and $n \in \mathbb{Z}$. Hence the lemma.

Proposition 3.9. $\left(\mathscr{H}^{j} p_{131}\right)\left(\mathscr{K}^{-N} r^{*}\right)\left(\mathcal{C V}_{1} \rrbracket \mathcal{V}_{2}\right) \in \mathcal{A}$ for any $j$ and $\mathcal{V}_{1}$, $\mathcal{V}_{2} \in \mathcal{A}$.

Proof. By Lemma 3.3(i) we may assume that $\mathrm{Gr}_{k}{ }^{W} C V_{i}=0$ for $k \neq n_{i}(i$ $=1,2)$. Then by Lemma 3.3 (ii) we have $\mathrm{Gr}_{k}{ }^{W}\left(\left(\mathcal{H}^{j} p_{13 !}\right)\left(\mathcal{H}^{-N} r^{*}\right)\left(\mathcal{C V}_{1} \backslash \mathcal{V}_{2}\right)\right)$ $=0$ for $k \neq n+j$ with $n=n_{1}+n_{2}-N$. Hence we have :

$$
\begin{aligned}
{\left[\left(\mathcal{H}^{j} p_{13 !}\right)\left(\mathcal{H}^{-N} r^{*}\right)\left(\mathcal{V}_{1} \rrbracket \mathcal{V}_{2}\right)\right] } & =\sum_{(w, \gamma, i) \in J_{j}} m_{w, \gamma, i}^{j} q^{i}\left[\mathcal{L}_{w} \rrbracket H_{\gamma}\right] \\
& =\sum_{(w, Y, i) \in J_{j}} m_{w, \gamma, i}^{j} q^{i}\left[H_{r}\right] C_{w}{ }^{\prime \prime},
\end{aligned}
$$

where $J_{j}=\left\{(w, \gamma, i) \in W \times \Gamma \times \boldsymbol{Z} \mid N+l(w)+n_{\gamma}+2 i=n+j\right\}$ and $m_{w, \gamma, i}^{j} \in \boldsymbol{Z}_{\geq 0}$. On the other hand we have :

$$
\sum_{j}(-1)^{j}\left[\left(\mathscr{H}^{j} p_{13 !}\right)\left(\mathcal{H}^{-N} r^{*}\right)\left(\mathcal{V}_{1} \rrbracket \mathcal{V}_{2}\right)\right]=\left[\mathcal{V}_{1}\right] \cdot\left[\mathcal{C} \mathcal{V}_{2}\right] \in H(W)
$$

by Lemma 3.8. Hence $m_{w, \gamma, i}^{j}=0$ for $\gamma \neq \gamma_{0}$ and the assertion is proved.

In consequence we have the following.

Theorem A. (i) The Grothendieck group $K(\mathcal{A})$ of the abelian category $\mathcal{A}$ is endowed with a $\mathbb{Z}\left[q, q^{-1}\right]$-algebra structure by:

$$
\left[\mathcal{V}_{1}\right] \cdot\left[\mathcal{C V}_{2}\right]=\sum_{j}(-1)^{j}\left[\left(\mathcal{H}^{j} p_{131}\right)\left(\mathcal{H}^{-N} r^{*}\right)\left(\mathcal{C V}_{1} \rrbracket \mathcal{V}_{2}\right)\right]
$$

and

$$
q^{n}[\mathcal{C V}]=[\mathcal{C V}(-n)]
$$


(ii) $K(\mathcal{A})$ is isomorphic to $H(W)$ as a $\mathbb{Z}\left[q, q^{-1}\right]$-algebra via the correspondence :

$$
\left[\mathcal{M}_{w}\right] \leftrightarrow(-1)^{l(w)} T_{w} \quad \text { and } \quad\left[\mathcal{L}_{w}\right] \leftrightarrow(-1)^{l(w)} \sum_{y \leq w} P_{y, w}(q) T_{y} .
$$

3.3. Let $K$ be a closed subgroup of $G$ which is either

(a) a Borel subgroup of $G$,

or

(b) a subgroup of $G^{\vartheta}$ containing $\left(G^{\vartheta}\right)^{0}$, where $\vartheta$ is an involutive automorphism of $G$.

Then it is known that the number of $K$-orbits on $X$ is finite and for any $x \in X$ the component gvoup $K^{x} /\left(K^{x}\right)^{0}$ of the stabilizer $K^{x}$ is isomovphic to $(\mathbb{Z} / 2 \mathbb{Z})^{N}$ for some $N \geqq 0$ (see [Ma],[LV]).

For a $K$-orbit $O$ on $X$ and a simple object $S$ of $\operatorname{Loc}(O, K)$, we set:

$$
\mathcal{L}(O, S)=\mathcal{L}(\bar{O}, X, S) \text { and } \mathcal{M}(O, S)=\mathscr{H}^{0} i_{1}\left(\Phi_{o}\left(H_{S}\right)\right),
$$

where $i: O \rightarrow X$ is the natural inclusion (see Section 1.5). Let $\mathcal{A}^{K}$ be the full subcategory of $\operatorname{MHM}(X, K)$ consisting of $\mathcal{V} \in M H M(X, K)$ such that for any $k \in \mathbb{Z} \mathrm{Gr}_{k}{ }^{W}(\mathcal{V})$ is a direct sum of the objects of the form $\mathcal{L}(O, S)(n)$ with $k=\operatorname{dim} O-2 n$. We define $p_{1}: X \times X \rightarrow X$ and $q: X \times X \rightarrow X \times X \times X$ by $p_{1}(a, b)=a$ and $q(a, b)=(a, b, b)$.

Theorem $\mathbf{A}^{\prime}$. (i) $\mathscr{M}(O, S) \in \mathcal{A}^{K}$.

(ii) Both of $\{[\mathcal{L}(O, S)] \mid(O, S)\}$ and $\{[\mathcal{M}(O, S)] \mid(O, S)\}$ are bases of $K\left(\mathcal{A}^{K}\right)$ over $\mathbb{Z}\left[q, q^{-1}\right]$.

(iii) For $\mathcal{Q} \in \mathcal{A}$ and $\Re \in \mathcal{A}^{K}$ we have $\left(\mathscr{H}^{j} q^{*}\right)(\mathcal{Q} \times \Re)=0$ for $j \neq-N$ and $\left(\mathcal{H}^{i} p_{11}\right)\left(\mathcal{H}^{-N} q^{*}\right)(\mathcal{Q} \backslash \Re) \in \mathcal{A}^{K}$ for any $i$.

(iv) An action of the $\mathbb{Z}\left[q, q^{-1}\right]$-algebra $K(\mathcal{A})$ on $K\left(\mathcal{A}^{K}\right)$ is defined by:

$$
[\mathcal{C V}] \cdot[\Re]=\sum_{j}(-1)^{j}\left[\left(\mathcal{H}^{j} p_{1^{1}}\right)\left(\mathcal{H}^{-N} q^{*}\right)(\mathcal{Q} 区 \Re)\right] .
$$

Hence $K\left(\mathfrak{A}^{K}\right)$ is an $H(W)$-module.

(v) When $K$ is of type (a) (hence a Borel subgroup $B$ ), $K\left(\mathcal{A}^{B}\right)$ is isomorphic to $K(\mathcal{A})$ as a left $H(W)$-module via the correspondence:

$$
\left[\mathcal{L}\left(X_{w}, \mathbb{Q}\right)\right] \leftrightarrow\left[\mathcal{L}_{w^{-1}}\right] \text { and }\left[\mathscr{M}\left(X_{w}, \mathbb{Q}\right)\right] \leftrightarrow\left[\mathscr{M}_{w^{-1}}\right] .
$$

Here $X_{w}$ is the Schubert cell $B w B / B$. 
(vi) When $K$ is of type (b), let $M$ be the $H(W)$-module constructed in [LV]. It has two free bases $\{\delta \mid \delta \in \mathscr{D}\}$ and $\left\{C_{\delta} \mid \delta \in \mathscr{D}\right\}$ over $\mathbb{Z}\left[q, q^{-1}\right]$, where $\mathscr{D}$ is the set of the pairs $(O, S)$ of $K$-orbits $O$ and simple objects $S$ of $\operatorname{Loc}(O$, $K)$. Then $K\left(\mathcal{A}^{K}\right)$ is isomorphic to $M$ as an $H(W)$-module via the correspondence :

$$
[\mathcal{M}(O, S)] \leftrightarrow(-1)^{\operatorname{dim} o} \delta \quad \text { and } \quad[\mathcal{L}(O, S)] \leftrightarrow(-1)^{\mathrm{dim} o} C_{\delta}
$$

with $\delta=(O, S)$.

The proof is similar to that of Theorem A.

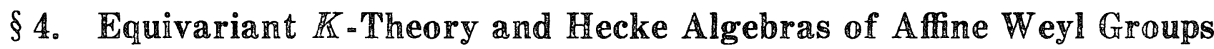

\subsection{Hecke algebras of affine Weyl groups}

Let $B$ be a Borel subgroup of $G$ and $T$ a maximal torus of $G$ contained in $B$. We choose an ordering on the root system so that the weights of $\operatorname{Lie}(G) / \operatorname{Lie}(B)$ are positive roots. The Weyl group $W\left(=N_{G}(T) / T\right)$ acts naturally on the weight lattice $P\left(=\operatorname{Hom}\left(B, \mathbb{C}^{*}\right)=\operatorname{Hom}\left(T, \mathbb{C}^{*}\right)\right)$. We denote the semidirect product $W \propto P$ by $W_{a}$ and call it the affine Weyl group of $G$.

When $G$ is an adjoint group, $W_{a}$ is a Coxeter group and the Hecke algebra $H\left(W_{a}\right)$ is defined. Besides the usual Iwahori-Matsumoto relation ([IM]), there is another presentation of $H\left(W_{a}\right)$ due to Bernstein. Let us recall Bernstein's description of $H\left(W_{a}\right)$. (It is also defined for general $G$.) Let $\alpha_{s}$ be the simple root corresponding to $s \in S$. The Hecke algebra $H\left(W_{a}\right)$ is a $\mathbb{Z}\left[q, q^{-1}\right]$-algebra which satisfies the following conditions $(h 1)$ $\sim(h 3)$.

(h1) $H\left(W_{a}\right)=H(W) \bigotimes_{Z_{\left[q, q^{-1}\right]}} \mathbb{Z}\left[q, q^{-1}\right][P] \quad$ as a $\mathbb{Z}\left[q, q^{-1}\right]$-module.

$(h 2) \quad H(W) \rightarrow H\left(W_{a}\right)(h \rightarrow h \otimes 1) \quad$ and $\quad \mathbb{Z}\left[q, q^{-1}\right][P] \rightarrow H\left(W_{a}\right)(u \rightarrow$ $1 \otimes u)$ are algebra homomorphisms.

We view $H(W)$ and $\mathbb{Z}\left[q, q^{-1}\right][P]$ as subalgebras of $H\left(W_{a}\right)$. The element of $\mathbb{Z}\left[q, q^{-1}\right][P]$ corresponding to $\lambda \in P$ is denoted by $\vartheta_{\lambda}$ when it is regarded as an element of $H\left(W_{a}\right)$.

(h3) $T_{s} \vartheta_{\lambda}=\vartheta_{s(\lambda)} T_{s}+(q-1) \frac{\vartheta_{\alpha_{s}}\left(\vartheta_{\lambda}-\vartheta_{s(\lambda)}\right)}{\vartheta_{\alpha_{s}}-1}$ for $s \in S$ and $\lambda \in P$. 


\subsection{A result of Ginsburg and $\mathbb{K}$ azhadan-Lusztigg}

For $x \in X$ let $B_{x}$ be the corresponding Borel subgroup and $\mathfrak{n}_{x}$ the Lie algebra of the unipotent radical of $B_{x}$. We consider the equivariant $K$ homology group $K^{C \times C^{*}}(Z)$ of the variety

$$
Z=\left\{(x, y, A) \in X \times X \times \text { Lie } G \mid A \in \mathfrak{n}_{x} \cap \mathfrak{n}_{y}\right\},
$$

where the action of $G \times \mathbb{C}^{*}$ is given by:

$$
(g, z) \cdot(x, y, A)=(g \cdot x, g \cdot y, z A d(g) A) .
$$

Since the representation ring $R C^{*}$ of $\mathbb{C}^{*}$ is identified with $\mathbb{Z}\left[q, q^{-1}\right]$ via $\left[z \rightarrow z^{2}\right] \leftrightarrow q^{2}, K^{G \times C^{*}}(Z)$ is a $\mathbb{Z}\left[q, q^{-1}\right]$-module. Ginsburg and KazhdanLusztig have defined an $\left(H\left(W_{a}\right), H\left(W_{a}\right)\right)$-bimodule structure on $K^{G \times C^{*}}(Z)$ and shown that it is isomorphic to the two-sided regular representation ([KL4], [Gi2]). We explain this slightly modifying the formulation of Ginsburg.

Since the dual space of $\operatorname{Lie}(G) / \operatorname{Lie}\left(B_{x}\right)$ is naturally identified with $\mathfrak{n}_{x}$ via the Killing form, the cotangent bundle $T^{*} X$ of $X$ is identified with the variety $\left\{(x, A) \in X \times \operatorname{Lie}(G) \mid A \in \mathfrak{n}_{x}\right\}$. Hence we can view $Z$ as a $G \times \mathbb{C}^{*}$. stable closed subvariety of $T^{*} X \times T^{*} X=\left\{\left(x, y, A, A^{\prime}\right) \mid A \in \mathfrak{n}_{x}, A^{\prime} \in n_{y}\right\}$ by $(x, y, A) \leftrightarrow(x, y, A, A)$. Let $p_{\imath}: T^{*} X \times T^{*} X \times T^{*} X \rightarrow T^{*} X \times T^{*} X$ and $p_{2}: T^{*} X \times T^{*} X \times T^{*} X \rightarrow T^{*} X$ be the projections and $p: T^{*} X \rightarrow X$ the cotangent bundle. It is easily seen from Lemma 2.1 and 2.2 that a $\mathbb{Z}\left[q, q^{-1}\right]$-module structure on $K^{G \times C^{*}}(Z)\left(=K^{G \times C^{*}}\left(Z, T^{*} X \times T^{*} X\right)\right)$ is given by :

$$
m_{1} \cdot m_{2}=p_{13 *}\left(p_{12} * m_{1} \otimes p_{23}{ }^{*} m_{2} \otimes p_{2}{ }^{*} p^{*}\left[\Omega_{X}\right]\right) .
$$

For $\lambda \in p$ let $O(\lambda)$ be the invertible $O_{x}$-module consisting of sections of the line bundle on $X$ with $G$-action such that the action of $B_{x}$ on the fiber at $x \in X$ is given by $\lambda$. For $s \in S$ we denote the closure of $\{(x, y, A) \in Z \mid$ $\left.(x, y) \in Y_{s}\right\}$ by $Z_{s}$. It is a $G$-equivariant vector bundle over $\bar{Y}_{s}$ via the natural projection $p_{s}: Z_{s} \rightarrow \bar{Y}_{s}$. Let $j: T^{*} X \rightarrow T^{*} X \times T^{*} X$ be the diagonal embedding and $j_{s}: Z_{s} \rightarrow T^{*} X \times T^{*} X$ the natural inclusion. We set:

$$
e(\lambda)=j_{*} p^{*}\left(\left[O(\lambda) \otimes \Omega_{X}{ }^{-1}\right]\right) \text { and } a_{s}=j_{s *} p_{s}{ }^{*}\left(\left[\Omega_{\bar{Y}}{ }^{s} / X \times X\right]\right)
$$

for $\lambda \in p$ and $s \in S$. They are elements of $K^{G \times c^{*}}(Z)=K^{G \times c^{*}}\left(Z, T^{*} X \times\right.$ $\left.T^{*} X\right)$. 
Theorem 4.1.([Gi, 2]). $K^{G \times c^{*}}(Z)$ is isomorphic to $H\left(W_{a}\right)$ as a $\boldsymbol{Z}\left[q, q^{-1}\right]$-algebra via the correspondence :

$$
q a_{s} \leftrightarrow-\left(T_{s}+1\right)(s \in S) \text { and } \quad e(-\lambda) \leftrightarrow \vartheta_{\lambda}(\lambda \in p) .
$$

4.3. Let $a$ be the involution on $T^{*} X \times T^{*} X$ given by $a\left(x, y, A, A^{\prime}\right)=$ $\left(x, y, A,-A^{\prime}\right)$. Since $\Lambda_{(X \times X, G)}=\left\{(x, y, A,-A) \in T^{*} X \times T^{*} X \mid A \in \mathfrak{n}_{x} \cap n_{y}\right\}$,

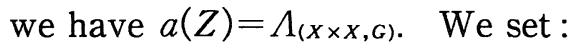

$$
\gamma=q^{N}\left(a^{*} \circ \mathrm{gr}\right): K(\mathcal{A}) \rightarrow K^{G \times C^{*}}(Z) .
$$

Theorem B. $\quad \gamma$ is a homomorphism of $\mathbb{Z}\left[q, q^{-1}\right]$-algebras. If we identify $K(\mathcal{A})$ and $K^{G \times c^{*}}(Z)$ with $H(W)$ and $H\left(W_{a}\right)$, respectively, then $\gamma$ coincides with the natural inclusion.

Let $K$ be a closed subgroup of $G$ which is either of type (a) or (b) in Section 3.3. Let $q_{i}: T^{*} X \times T^{*} X \rightarrow T^{*} X$ be the obvious projections $(i=1$ $2)$. It is easily seen that an action of $K^{G \times C^{*}}(Z)$ on $K^{K \times c^{*}}\left(\Lambda_{(X, K)}\right)$ is defined by :

$$
m \cdot n=q_{1 *}\left(m \otimes q_{2}{ }^{*} n \otimes q_{2}{ }^{*} p^{*}\left[\Omega_{X}\right]\right)\left(m \in K^{G \times C^{*}}(Z), n \in K^{K \times c^{*}}\left(\Lambda_{(X, K)}\right)\right) .
$$

Especially, $K^{K \times C^{*}}\left(\Lambda_{(X, K)}\right)$ is an $H(W)$-module.

Theorem $\mathbf{B}^{\prime} . \mathrm{gr}: K\left(\mathcal{A}^{K}\right) \rightarrow K^{K \times C^{*}}\left(\Lambda_{(X, K)}\right)$ is a homomorphism of $H(W)$-modules.

We give the proof of Theorem B. Theorem $\mathrm{B}^{\prime}$ is proved similarly.

Proof of Theorem B. Let $\sigma: Y_{e} \rightarrow T^{*} Y_{e}$ be the zero section. Since $\mathcal{L}_{e}=i_{e *}\left(\mathcal{L}_{Y_{e}}\right)$ and $\operatorname{gr}\left(\mathcal{L}_{Y_{e}}\right)=\sigma_{*}\left(O_{Y_{e}}\right)$, we see from Lemma 2.5 that $\gamma\left(\left[\mathcal{L}_{e}\right]\right)=$ $e(0)$. Similarly we have $\gamma\left(\left[\mathcal{L}_{s}\right]\right)=q a_{s}$ for $s \in S$. Hence it is sufficient to show $\gamma\left(\left[\mathcal{L}_{s}\right] \cdot m\right)=q a_{s} \cdot \gamma(m)$ for $m \in K(\mathcal{A})$. We set $u_{i}=q_{i} \circ j_{s}$ for $i=1,2$. It is easily seen from Lemma 2.1 and Lemma 2.2 that:

$$
a_{s} \cdot n=\left(u_{1} \times 1\right)_{*}\left(u_{2} \times 1\right)^{*}\left(n \otimes(p \times p)^{*}\left(\left[\Omega_{X / X} \times \bigotimes O_{X}\right]\right)\right)
$$

for $n \in K^{G \times c^{*}}(Z)$. On the other hand we have $\left[\mathcal{L}_{s}\right] \cdot m=-\left(\pi_{s} \times 1\right)^{*}\left(\pi_{s} \times\right.$ $1)_{*}(m)$. Thus we can see easily from Lemma 2.4 and 2.5 that:

$$
\gamma\left(\left[\mathcal{L}_{s}\right] \cdot m\right)=q\left(u_{1} \times 1\right)_{*}\left(u_{2} \times 1\right)^{*}\left(\gamma(m) \otimes(p \times p) *\left(\left[\Omega_{X / X} \backslash \bigotimes O_{X}\right]\right)\right) .
$$

Hence the assertion is proved.

Remark. Theorem B and Theorem B' are generalization of the results in $[\mathrm{KT}]$ and $[\mathrm{Ta}]$. 


\section{$\S 5$. Good Filtrations of $U(\mathrm{~g})$-Modules Associated to Hodge Modules}

5.1. We denote the enveloping algebra of $\mathrm{g}=\operatorname{Lie}(G)$ by $U(\mathrm{~g})$.

$\mathrm{By}[\mathrm{BeB}]$ the category of coherent $D_{X}$-modules is equivalent to the category of finitely generated $U(\mathrm{~g})$-modules with trivial central character. The $U(\mathrm{~g})$-module corresponding to a coherent $D_{x}$-module $\mathcal{M}$ is $\Gamma(X, \mathcal{M})$, the space of its global sections. Note that $H^{2}(X, \mathscr{M})=0$ for $i>0([\mathrm{BeB}])$. When a good filtration $F$ of a coherent $D_{X}$-module $\mathcal{M}$ is given (for example when $\mathcal{M}$ is an underlying $D_{x}$-module of a Hodge module), the corresponding $U(\mathrm{~g})$-module $M=\Gamma(X, \mathcal{M})$ is equipped with a good filtration via $F_{p}(M)$ $=\Gamma\left(X, F_{p} \mathcal{M}\right) . \quad$ A good filtration of a finitely generated $U(\mathrm{~g})$-module is defined similarly to the case of a coherent $D$-module using the order filtration of $U(\mathrm{~g})$. Let $M F(\mathrm{~g})$ be the category consisting of pairs $(M, F)$ of finitely generated $U(\mathrm{~g})$-modules $M$ with trivial central character and their good filtrations $F$. By the above arguments we have a functor :

$$
\Gamma F: M H M(X) \rightarrow M F(\mathrm{~g}) .
$$

The category $M F(\mathrm{~g})$ is not an abelian category but an exact category. A sequence :

$$
\left[\cdots \rightarrow\left(M_{i-1}, F\right) \rightarrow\left(M_{i}, F\right) \rightarrow\left(M_{i+1}, F\right) \rightarrow \cdots\right]
$$

in $M F(g)$ is exact if and only if the associated graded sequence :

$$
\left[\cdots \rightarrow \mathrm{Gr}^{F} M_{i-1} \rightarrow \mathrm{Gr}^{F} M_{2} \rightarrow \mathrm{Gr}^{F} M_{i+1} \rightarrow \cdots\right]
$$

is exact in the abelian category of $\operatorname{Gr} U(\mathrm{~g})(=S(\mathrm{~g}))$-modules.

It is natural to ask whether $\Gamma F$ is an exact functor. Hence we are led to the following :

Question. Is it true that

$$
\text { (B) } \quad H^{2}\left(X, F_{p} \mathscr{M}\right)=0 \quad(i>0, p \in \mathbb{Z})
$$

for $C=(\mathscr{M}, F, K, W) \in M H M(X)$ ?

Similar problems are treated in [BoB].

By the exact sequence $\left[0 \rightarrow F_{p-1} \mathscr{M} \rightarrow F_{p} \mathscr{M} \rightarrow \mathrm{Gr}_{p}{ }^{F} \mathscr{M} \rightarrow 0\right]$ we see easily that $(B)$ is equivalent to : 


$$
\left(B^{\prime}\right) \quad H^{i}\left(X, \mathrm{Gr}^{F} \mathcal{M}\right)=0 \quad(i>0)
$$

and if this is true, then we have $\operatorname{Gr}^{F} M=\Gamma\left(X, \operatorname{Gr}^{F} \mathcal{M}\right)$ for $(M, F)=\Gamma F(\mathcal{V})$ and the functor $\Gamma F$ is exact.

Remark. Kashiwara has proved $(B)$ for $X=\mathbb{P}^{n}$ using Saito's Kodaira vanishing theorem ([Sa3]).

5.2. Identifying the cotangent bundle $T^{*} X$ with $\left\{(x, f) \in X \times g^{*} \mid\right.$ $\left.f\left(\operatorname{Lie}\left(B_{x}\right)\right)=0\right\}$ we define $\tau: T^{*} X \rightarrow \mathrm{g}^{*}$ by $\tau(x, f)=f$ (the moment map). We fix a Borel subgroup $B$ of $G$. It is easily seen that $\Lambda_{(X, B)}=\tau^{-1}\left(\mathfrak{b}^{\perp}\right)$ where $\mathfrak{b}$ is the Lie algebra of $B$ and $\mathfrak{b}^{\perp}=\left\{f \in g^{*} \mid f(\mathfrak{b})=0\right\} . \quad \mathfrak{b}^{\perp}$ can be identified with $[G, G]$ via the Killing form. Consider the maps:

$$
\begin{aligned}
K\left(\mathcal{A}^{B}\right) & \stackrel{\mathrm{gr}}{\longrightarrow} K^{B \times C^{*}}\left(\Lambda_{(X, B)}, T^{*} X\right) \\
& \stackrel{\tau^{*}}{\longrightarrow} K^{B \times C^{*}}\left(\mathfrak{G}^{\perp}\right)=K^{B \times C^{*}}\left(\mathfrak{G}^{\perp}, \mathrm{g}^{*}\right) .
\end{aligned}
$$

$K^{B \times C^{*}}\left(\mathfrak{b}^{\perp}\right)$ can be identified with the representation ring $R_{B \times C^{*}}=\mathbb{Z}\left[q, q^{-1}\right][P]$ $=\bigoplus_{\mu \in p} \mathbb{Z}\left[q, q^{-1}\right] e^{\mu}$ via the Thom isomorphism $\bar{p}^{*}: R_{B \times C^{*}}\left(=K^{B \times C^{*}}(p t)\right) \rightarrow K^{B \times}$ $c^{*}\left(\mathfrak{b}^{\perp}\right)$, where $\bar{p}: \mathfrak{b}^{\perp} \rightarrow p t$.

Lemma 5.1. ([Lu], see also Kato's proof given in [KL4]).

An action of the Hecke algebra $H\left(W_{a}\right)$ on $\mathbb{Z}\left[q, q^{-1}\right][P]$ is given by:

$$
\begin{aligned}
& T_{s} \cdot x=\frac{x-s(x) e^{-2 \alpha}}{e^{\alpha}-1}-q \frac{x-s(x) e^{\alpha}}{e^{\alpha}-1} \quad(s \in S), \\
& \vartheta_{\lambda} \cdot x=e^{-\lambda} x \quad(\lambda \in p),
\end{aligned}
$$

where $\alpha$ is the simple root corresponding to $s \in S$.

Proposition 5.2. For $w \in W$ we have:

$$
\tau_{*}\left(\operatorname{gr}\left(\left[\mathcal{L}\left(\bar{X}_{w}, X\right)\right]\right)\right)=q^{-N} C_{w}{ }^{\prime \prime} \cdot e^{2 \rho}
$$

in $K^{B \times C^{*}}\left(\mathfrak{b}^{\perp}\right)=\mathbb{Z}\left[q, q^{-1}\right][P]$, where $\rho$ is the half of the sum of the positive roots.

Although $\rho$ is not necessarily an element of $P, 2 \rho$ and $w \rho+\rho$ for $w \in$ $W$ are elements of $P$.

The proof of Proposition 5.2 will be given in Section 5.3. 
Let $\left(L_{w}, F\right)=\Gamma F\left(\mathcal{L}\left(\bar{X}_{w}, X\right)\right)$. It is known that $L_{w}$ is the irreducible lowest weight module with lowest weight $w \rho+\rho$ ([BK], $[\mathrm{BeB}])$. Note that we have chosen the ordering on the root system so that the set of positive roots $\Delta^{+}$coincides with the weights in $\mathrm{g} / \mathfrak{b}$.

Defimition. For a finitely generated $U(\mathrm{~g})$-module $M$ with $B$-action and a $B$-stable good filtration $F$ of $M$ we define the ' $q$-character' $\operatorname{ch}_{q}(M, F)$ of $(M, F)$ by :

$$
\operatorname{ch}_{q}(M, F)=\sum_{j \in Z} \operatorname{ch}\left(\mathrm{Gr}_{j}{ }^{F} M\right) q^{-j} \in \mathbb{Z}[P]\left(\left(q^{-1}\right)\right) .
$$

Here $\operatorname{ch}\left(\mathrm{Gr}_{j}{ }^{F} M\right) \in \mathbb{Z}[P]$ is the character of the $B$-module $\mathrm{Gr}_{j}{ }^{F} M$.

Corollary 5.3. If the condition $(B)$ holds for $C \mathcal{V}=\mathcal{L}\left(\bar{X}_{w}, X\right)$, then we have:

$$
\operatorname{ch}_{q}\left(L_{w}, F\right)=\frac{q^{-N} C_{w}{ }^{\prime \prime} \cdot e^{2 \rho}}{\prod_{\alpha \in \Delta^{+}}\left(1-q^{-1} e^{\alpha}\right)} .
$$

Proof. In general for $\mathcal{C}=(\mathcal{M}, F, K, W) \in M H M(X)$ we have

$$
\begin{aligned}
\mathbb{R} \Gamma\left(X, \mathrm{Gr}^{F} \mathscr{M}\right) & =\mathbb{R} \Gamma\left(X, \mathbb{R} p_{*}(\operatorname{gr}(\mathcal{V}))\right) \\
& =\mathbb{R} \Gamma\left(T^{*} X, \operatorname{gr}(\mathcal{V})\right) \\
& =\mathbb{R} \Gamma\left(\mathrm{g}^{*}, \mathbb{R} \tau_{*}(\operatorname{gr}(\mathcal{V}))\right) .
\end{aligned}
$$

Hence when the condition $(B)$ holds for $V$, we have :

$$
\begin{aligned}
\operatorname{Gr}^{F} M & =\Gamma\left(X, \operatorname{Gr}^{F} \mathscr{M}\right)\left(=\mathbb{R} \Gamma\left(X, \mathrm{Gr}^{F} \mathcal{M}\right)\right) \\
& =\Gamma\left(\mathrm{g}^{*}, \tau_{*}(\operatorname{gr}(\mathcal{C V}))\right)\left(=\mathbb{R} \Gamma\left(\mathrm{g}^{*}, \mathbb{R} \tau_{*}(\operatorname{gr}(\mathcal{V}))\right)\right)
\end{aligned}
$$

for $(M, F)=\Gamma F(C V)$. Therefore

$$
\operatorname{ch}_{q}(M, F)=\frac{\tau_{*}(\operatorname{gr}([\mathcal{C}]))}{\prod_{\alpha \in \Delta_{+}}\left(1-q^{-1} e^{\alpha}\right)},
$$

and the assertion follows from Proposition 5.2. Here $\left(\prod_{\alpha \in \Delta^{+}}\left(1-q^{-1} e^{\alpha}\right)\right)^{-1}=$ $\prod_{\alpha \in \Delta^{+}}\left(\sum_{k \geq 0} q^{-k} e^{k \alpha}\right)$ appears as the character of the $B \times \mathbb{C}^{*}$-module $\Gamma\left(\mathfrak{b}^{\perp}, O_{b^{-}}\right)$.

\subsection{Proof of Proposition 5.2}

The arguments below are inspired by $[\mathrm{BoB}]$.

We first give some relations of $\mathcal{A}$ and $\mathcal{A}^{B}$. Let $x_{0} \in X$ be the point corresponding to $B$. We define $k: X \rightarrow X \times X$ by $k(x)=\left(x, x_{0}\right)$. 
Lemma 5.4. (i) $\left(\mathscr{H}^{j}{ }^{j}{ }^{*}\right)(\mathcal{C V})=0$ for $\mathscr{C V} \in M H M(X \times X, G)$ and $j \neq-N$.

(ii) $\left(\mathscr{H}^{-N} k^{*}\right)\left(\mathcal{L}_{w}\right)=\mathcal{L}\left(\bar{X}_{w-1}, X\right)$ for $w \in W$.

(iii) $\mathscr{T}^{-N} k^{*}$ induces exact functors :

$$
\operatorname{MHM}(X \times X, G) \rightarrow \operatorname{MHM}(X, B) \text { and } \mathcal{A} \rightarrow \mathcal{A}^{B} .
$$

Proof. (i) is shown in the proof of Lemma 3.3. Choose a Borel subgroup which is opposit to $B$ and denote its unipotent radical by $U$. We define $\varphi: X \times U \rightarrow X \times X$ by $\varphi(x, u)=\left(u \cdot x, u \cdot x_{0}\right)$. It is an open immeresion. By the proof of Lemma 3.3 we have $\varphi^{*} \mathcal{V} \simeq\left(\mathcal{H}^{-N} k^{*}\right)(\mathcal{C V}) \bigotimes \mathcal{L}_{U}$ for $\mathcal{V} \in M H M(X \times X, G)$. Since $\varphi^{-1}\left(Y_{w}\right)=X_{w^{-1}} \times U$, we have :

$$
\begin{aligned}
\mathcal{L}\left(\bar{X}_{w^{-1}}, X\right) \rrbracket \mathcal{L}_{U} & \simeq \mathcal{L}\left(\bar{X}_{w^{-1}} \times U, X \times U\right) \\
& =\varphi^{*} \mathcal{L}_{w} \\
& =\left(\mathcal{H}^{-N} k^{*}\right)\left(\mathcal{L}_{w}\right) \rrbracket \mathcal{L}_{U},
\end{aligned}
$$

and (ii) is proved. (iii) is a consequence of (i) and (ii).

We identify $\left(T^{*} X\right)_{x_{0}}$, the fiber of $T^{*} X$ at $x_{0}$, with $\mathfrak{n}=\left[\mathfrak{b} x_{0}, \mathfrak{b} x_{0}\right]$. Let $\varpi: \mathrm{T}^{*} X \times \mathfrak{n}\left(=T^{*} X \times\left(T^{*} X\right)_{x_{0}}\right) \rightarrow T^{*}(X \times X)\left(=T^{*} X \times T^{*} X\right)$ be the inclusion and $\rho: T^{*} X \times \mathfrak{n} \rightarrow T^{*} X$ the projection. Identifying $T^{*} X$ with $\{(x$, $\left.A) \in X \times \mathfrak{g} \mid A \in \mathfrak{n}_{x}\right\}$ we have :

$$
\begin{aligned}
\Lambda_{(X \times X, G)} & =\left\{(x, y, A,-A) \in X \times X \times \mathrm{g} \times \mathrm{g} \mid A \in \mathfrak{n}_{x} \cap \mathfrak{n}_{y}\right\}, \\
\Lambda_{(X, B)} & =\left\{(x, A) \in X \times \mathrm{g} \mid A \in \mathfrak{n}_{x} \cap \mathfrak{n}\right\} .
\end{aligned}
$$

We define subvarieties $\Lambda^{+}$and $\Lambda^{-}$of $T^{*} X \times \mathfrak{n}$ by :

$$
\Lambda^{ \pm}=\left\{((x, A), \pm A) \mid A \in \mathfrak{n}_{x} \cap \mathfrak{n}\right\} .
$$

Since $\rho$ induces an isomorphism $\Lambda^{-} \leadsto \Lambda_{(X, B)}$, and since $\varpi^{-1}\left(\Lambda_{(X \times X, G)}\right)=\Lambda^{-}$, we have the natural maps:

$$
\begin{aligned}
& \varpi^{*}: K^{G \times c^{*}}\left(\Lambda_{(X \times X, G)}, T^{*}(X \times X)\right) \rightarrow K^{B \times C^{*}}\left(\Lambda^{-}, T^{*} X \times \mathfrak{n}\right) \\
& \rho_{*}: K^{B \times c^{*}}\left(\Lambda^{-}, T^{*} X \times \mathfrak{n}\right) \rightarrow K^{B \times c^{*}}\left(\Lambda_{(X, B)}, T^{*} X\right) .
\end{aligned}
$$

Lemma 5.5. For $\mathcal{V} \in M H M(X \times X, G)$ we have:

$$
\rho_{*} \varpi^{*}(\operatorname{gr}([\mathcal{C V}]))=\operatorname{gr}\left(\left[\left(\mathcal{H}^{-N} k^{*}\right)(\mathcal{C V})\right]\right) .
$$

This follows from the fact that $\varphi^{*} \mathcal{C V}=\left(\mathscr{H}^{-N} k^{*}\right)(\mathcal{C V}) \bigotimes \mathcal{L}_{U}$ for $\mathcal{V} \in$ $\operatorname{MHM}(X \times X, G)$ in the notation of the proof of Lemma 5.4. Details are left to the readers. 
Consider the following commutative diagram.

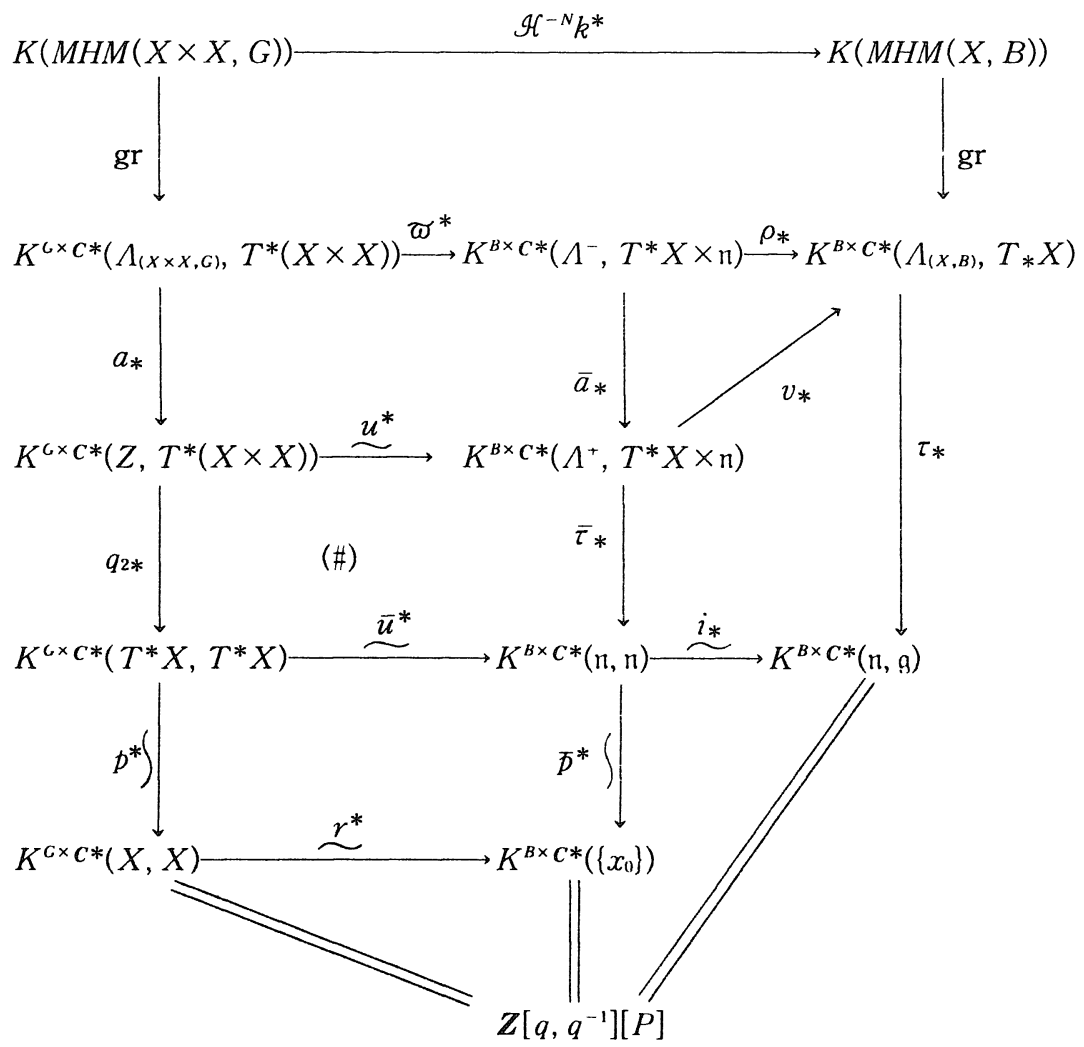

Here $u, \bar{u}, r, i$ are natural inclusions and

$$
\begin{aligned}
& a\left(x, y, A, A^{\prime}\right)=\left(x, y, A,-A^{\prime}\right), \\
& \bar{a}\left((x, A), A^{\prime}\right)=\left((x, A),-A^{\prime}\right), \\
& v\left((x, A), A^{\prime}\right)=(x, A), \\
& \bar{\tau}\left((x, A), A^{\prime}\right)=A^{\prime} .
\end{aligned}
$$

Note that $q_{2} \mid Z$ and $\bar{\tau} \mid \Lambda^{+}$are projective morphisms. The commutativity of (\#) follows easily from Lemma 2.1 and Lemma 2.2 since $\bar{u}$ is a closed immersion and $q_{2}$ is smooth.

By Lemma 5.4 we have $\tau_{*}\left(\operatorname{gr}\left(\left[\mathcal{L}\left(\bar{X}_{w}, X\right)\right]\right)\right)=q_{2 *}\left(a_{*}(\operatorname{gr}(\mathcal{L}\right.$ $\left.\left.\left.w^{-1}\right)\right)\right) q_{1 *}\left(a_{*}\left(\operatorname{gr}\left(\left[\mathcal{L}_{w}\right]\right)\right)\right)$ in $\mathbb{Z}\left[q, q^{-1}\right][P]$. The last equality follows from an easy calculation involving the G-equivariant automorphism of $X \times X$ given by $(x, y) \rightarrow(y, x)$. By Theorem $4.1 K^{G \times C^{*}}(Z)$ is identified with $H\left(W_{a}\right)$. Define $F: H\left(W_{a}\right) \rightarrow \mathbb{Z}\left[q, q^{-1}\right][P]$ by the commutativity of : 


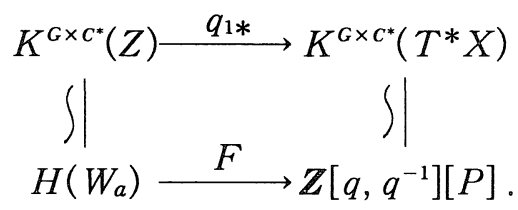

By Theorem $A$ and Theorem $B$ we have $\tau_{*}\left(\operatorname{gr}\left(\left[\mathcal{L}\left(\bar{X}_{w}, X\right)\right]\right)\right)=q^{-N} F\left(C_{w}{ }^{\prime \prime}\right)$. Hence Proposition 5.2 is a consequence of the following :

Lemma 5.6. (i) $F$ is a homomorphism of $H\left(W_{a}\right)$-modules. Here the $H\left(W_{a}\right)$-module structure of $H\left(W_{a}\right)$ is given by the left multiplication and that of $\mathbb{Z}\left[q, q^{-1}\right][P]$ is the one given in Lemma 5.1 .

(ii) $F(1)=e^{2 \rho}$.

Proof. It is easily seen that a $K^{G \times C^{*}}(Z)$-module structure on $K^{G \times C^{*}}$ $\left(T^{*} X\right)$ is defined by :

$$
\begin{aligned}
& h \cdot m=q_{1 *}\left(h \otimes q_{2}{ }^{*}\left(m \otimes p^{*}\left(\left[\Omega_{X}\right]\right)\right)\right) \\
&\left(h \in K^{G \times C^{*}}\left(Z, T^{*}(X \times X)\right), \quad m \in K^{G \times C^{*}}\left(T^{*} X, T^{*} X\right)\right) .
\end{aligned}
$$

By a standard argument we see that $q_{1 *}$ is a homomorphism of $K^{G \times C^{*}}(Z)$ modules and that the $K^{G \times C^{*}}(Z)\left(=H\left(W_{a}\right)\right)$-module structure on $K^{G \times c^{*}}$ $\left(T^{*} X\right)\left(=\mathbb{Z}\left[q, q^{-1}\right][P]\right)$ coincides with the one given in Lemma 5.1. (i) is proved. (ii) is a consequence of $q_{1 *}(e(0))=p^{*}\left(\left[\Omega_{X}{ }^{-1}\right]\right)$.

\section{References}

[BeB] Beilinson, A. and Bernstein, J., Localisation de g-modules, Comptes Rendus, 292 (1981), 15-18.

[BBD] Beilinson, A., Bernstein, J. and Deligne, P., Faisceaux pervers, Astérisque, 100 (1983).

[Be] Bernstein, J., Algebraic theory of $D$-modules, preprint (1983).

[BoB] Borho, W. and Brylinski, J. -L., Differential operators on homogeneous spaces III, Invent. Math., 86 (1985), 1-68.

[Br] Brylinski, J. -L., Modules holonomes à singularités regulières et filtration de Hodge II, Astérisque, 101-102 (1983), 75-117.

[BK] Brylinski, J. -L. and Kashiwara, M., Kazhdan-Lusztig conjecture and holonomic systems, Invent. Math., 64 (1981), 387-410.

[D1] Deligne, P., Equations différentielles à points singuliers réguliers, Springer Lecture Notes in Math., 163 (1970).

[D2] - P., Théorie de Hodge, I, Actes du Congrés international des Mathématiciens (Nice, 1970) 425-430, II et III, Publ. Math. IHES, 40 (1971), 5-57 et 44 (1974), 5-77.

[G1] Ginsburg, V., Lagrangean construction for representations of Hecke algebras, Adv. Math. 63 (1987), 100-112.

[G2] — - V., Deligne-Langlands conjecture and representations of affine Hecke algebras, 
preprint (1985).

[GM] Goresky, M. and MacPherson, R., Intersection homology II, Invent. Math., 72 (1983), 77 129 .

[I] Iwahori, N., On the structure of the Hecke ring of a Chevalley group over a finite field, J. Fac. Sci. Univ. Tokyo Sec 1A, 10 (1964), 215-236.

[IM] Iwahori, N. and Matsumoto, H., On some Bruhat decompositions and the structures of the Hecke rings of $p$-adic Chevalley groups, Publ. IHES, 25 (1965), 5-48.

[K] Kashiwara, M., The Riemann-Hilbert problem for holonomic systems, Publ. RIMS, 20 (1984), 319-365.

[KK] Kashiwara, M. and Kawai, T., On the holonomic systems of microdifferential equations. III, Publ. RIMS, 17 (1981), 813-979.

[KT] Kashiwara, M. and Tanisaki, T., The characteristic cycles of holonomic systems on a flag manifold-related to the Weyl group algebra, Invent. Math., 77 (1984), 185-198.

[KL1] Kazhdan, D. and Lusztig, G., Representations of Coxeter groups and Hecke algebras, Invent. Math., 53 (1979), 165-184.

[KL2] - Schubert varieties and Poincaré duality, Proc. Symp. in Pure Math., 36 (1980). 185203.

[KL3] - Equivariant $K$-theory and representations of Hecke algebras II, Invent. Math., 80 (1985), 209-231.

[KL4] — Proof of Deligne-Langlands conjecture for Hecke algebras, Invent. Math., 87 (1987), 153-215.

[La] Laumon, G., Sur la catégorie dérivée des D-Modules filtrés, Springer Lecture Notes in Math., 1016 (1984), 151-237.

[Lu] Lusztig, G., Equivariant $K$-theory and representations of Hecke algebras, Proc. Amer. Math Soc., 94 (1985), 337-342.

[LV] Lusztig, G. and Vogan, D., Singularities of closures of $K$-orbits on flag manifolds, Invent. Math., 71 (1983), 365-379.

[Ma] Matsuki. T., The orbits of affine symmetric spaces under the action of minimal parabolic subgroups, J. Math. Soc. Japan 31 (1979), 331-357.

[Me1] Mebkhout, Z., Une équivalence de catégories, Comp. Math., 51 (1984), 51-62.

[Me2] —- Une autre équivalence de catégories, Comp. Math., 51 (1984), 63-88.

[Sa1] Saito, M., Hodge structure via filtered $D$-modules, Astérisque, 130 (1985), 342-351.

[Sa2] —- Modules de Hodge plarisables, preprint RIMS-553 (1986).

[Sa3] - Mixed Hodge Modules (announcement), Proc. Japan Acad., 62 (1986), 360-363.

[Sa4] - On the derived category of mixed Hodge Modules, Proc. Japan Acad., 62 (1986), 364-366.

[Sa5] - Mixed Hodge Modules, in preparation.

[Sp] Springer, T. A., Quelque applications de la cohomologie d'intersection, Séminaire Bourbaki, exposé 589, Astérisque, 92-93 (1982), 249-273.

[Ta] Tanisaki, T., Holonomic systems on a flag variety associated to Harish-Chandra modules and representations of a Weyl group. Adv. Studies in Pure Math., 6 (1985), 139. 154.

[Th] Thomason, R., Algebraic $K$-theory of group scheme actions, to appear in Proc. Topol. Conf. in honor J. Moore, Princeton (1983). 
